\title{
Cassini RADAR Sequence Planning and Instrument Performance
}

\author{
Richard D. West, Yanhua Anderson, Rudy Boehmer, Leonardo Borgarelli, Philip Callahan, Charles Elachi, \\ Yonggyu Gim, Gary Hamilton, Scott Hensley, Michael A. Janssen, William T. K. Johnson, \\ Kathleen Kelleher, Ralph Lorenz, Steve Ostro, Member, IEEE, Ladislav Roth, Scott Shaffer, \\ Bryan Stiles, Steve Wall, Lauren C. Wye, and Howard A. Zebker, Fellow, IEEE
}

\begin{abstract}
The Cassini RADAR is a multimode instrument used to map the surface of Titan, the atmosphere of Saturn, the Saturn ring system, and to explore the properties of the icy satellites. Four different active mode bandwidths and a passive radiometer mode provide a wide range of flexibility in taking measurements. The scatterometer mode is used for real aperture imaging of Titan, high-altitude (around $20000 \mathrm{~km}$ ) synthetic aperture imaging of Titan and Iapetus, and long range (up to $700000 \mathrm{~km}$ ) detection of disk integrated albedos for satellites in the Saturn system. Two SAR modes are used for high- and medium-resolution (300-1000 m) imaging of Titan's surface during close flybys. A high-bandwidth altimeter mode is used for topographic profiling in selected areas with a range resolution of about $35 \mathrm{~m}$. The passive radiometer mode is used to map emission from Titan, from Saturn's atmosphere, from the rings, and from the icy satellites. Repeated scans with differing polarizations using both active and passive data provide data that can usefully constrain models of surface composition and structure. The radar and radiometer receivers show very good stability, and calibration observations have provided an absolute calibration good to about $1.3 \mathrm{~dB}$. Relative uncertainties within a pass and between passes can be even smaller. Data are currently being processed and delivered to the planetary data system at quarterly intervals one year after being acquired.
\end{abstract}

Index Terms-Cassini, RADAR, radiometer, synthetic aperture radar (SAR).

\section{INTRODUCTION}

$\mathbf{T}$ HE Cassini-Huygens mission to Saturn is the largest planetary mission ever mounted with joint participation by the National Aeronautics and Space Administration (NASA),

Manuscript received February 12, 2008; revised May 7, 2008. First published February 6, 2009; current version published May 22, 2009. This work was performed at the JPL, California Institute of Technology, under contract with the NASA.

R. D. West, Y. Anderson, R. Boehmer, P. Callahan, C. Elachi, Y. Gim, G. Hamilton, S. Hensley, M. A. Janssen, W. T. K. Johnson, K. Kelleher, S. Ostro, L. Roth, S. Shaffer, B. Stiles, and S. Wall are with the Jet Propulsion Laboratory, California Institute of Technology, Pasadena, CA 91109 USA (e-mail: richard.west@jpl.nasa.gov; yanhua.anderson@jpl.nasa.gov; rboehmer@jpl.nasa.gov; phil.callahan@jpl.nasa.gov; charles.elachi@jpl.nasa. gov; yonggyu.gim@jpl.nasa.gov; gary.hamilton@jpl.nasa.gov; scott.hensley@ jpl.nasa.gov; mike.janssen@jpl.nasa.gov; wtk.johnson@jpl.nasa.gov; kathleen. kelleher@jpl.nasa.gov; steve.ostro@jpl; ladislav.roth@jpl.nasa.gov; scott. shaffer@jpl.nasa.gov; bryan.stiles@jpl.nasa.gov; steve.wall@jpl.nasa.gov).

L. Borgarelli is with the Thales Alenia Space Italia, 00131 Rome, Italy (e-mail: 1.borgarelli@ roma.alespazio.it).

R. Lorenz is with the Applied Physics Laboratory, Johns Hopkins University, Baltimore, MD 21218 USA (e-mail: ralph.lorenz@ jhuapl.edu).

L. C. Wye and H. A. Zebker are with Stanford University, Stanford, CA 94305 USA (e-mail: lcwye@stanford.edu; zebker@stanford.edu).

Digital Object Identifier 10.1109/TGRS.2008.2007217 the European Space Agency, and the Italian Space Agency (ASI). Scientists and engineers from 17 different countries have worked on the Cassini spacecraft and the Huygens probe. The spacecraft was launched on October 15, 1997, and then embarked on a seven-year cruise out to Saturn with flybys of Venus, the Earth, and Jupiter. The spacecraft entered Saturn orbit on July 1, 2004 with a successful orbit insertion burn. This marked the start of an intensive four-year primary mission full of remote sensing observations by a dozen instruments carried by the orbiter, and the successful landing of the Huygens probe on Titan in January of 2005. One part of the remote sensing suite carried by the spacecraft is the Cassini RADAR, a multimode Ku-band $(13.8 \mathrm{GHz}, 2.2-\mathrm{cm}$ wavelength) radar/radiometer instrument that uses the highgain communications antenna to probe Titan and other targets of interest. This paper describes the science objectives, data collection strategies, calibration, and processing issues for the radar instrument. The discussion here complements and updates the discussion in a previous report [1].

\section{Science Objectives ANd Tour Design}

After arriving in Saturn orbit, the spacecraft started a carefully planned tour of the Saturn system. The prime mission tour consists of 67 orbits of Saturn with 45 Titan flybys, which are used as gravitational slingshots to direct the spacecraft along the planned tour trajectory. Titan flybys are also coveted opportunities to study Titan itself from a close distance. The 45 Titan flybys are divided among many different observation types including optical and infrared imaging and spectroscopy, gravity studies, occultation observations, in situ atmospheric observations, and radar observations.

The overall science objectives for the Cassini RADAR are covered in [1, Sec. 2]. In the years leading up to the start of the Saturn tour, a detailed science operation plan (SOP) was put together by the Cassini Project Science Team to address the science objectives of all its instruments. The Cassini spacecraft does not have a scan platform so it is not possible to point the optical remote sensing instruments and the radar at the same target at the same time. The radar observes with the highgain communications antenna that is pointed along the spacecraft $-Z$-axis. The optical remote sensing instruments are pointed along the $-Y$-axis while the inertial neutral mass spectrometer (INMS) is pointed along the $-X$-axis. The fixed instrument orientations and limited flyby durations have led to a complex choreographed series of observations. The current plan for the four-year tour has 145 planned radar observations. 
TABLE I

RADAR OBSERVATION SUMMARY FOR THE PRIME MISSION (2004-2008)

\begin{tabular}{|c|c|c|}
\hline Obs. Type & Radar Modes & No. Planned \\
\hline Imaging Titan Flybys & RAD,SCAT,ALT,SAR & 18 \\
Ride-Along Titan Flybys & RAD,SCAT,ALT,SAR & 3 \\
Non-imaging Titan Flybys & RAD,SCAT & 3 \\
Distant Titan Radiometry & RAD & 44 \\
Distant Titan Scatterometry & RAD,SCAT & 8 \\
Icy Satellite Scatterometry & RAD,SCAT & 38 \\
Saturn Radiometry & RAD & 5 \\
Rings Radiometry & RAD & 12 \\
Cosmic Source Scans & RAD & 8 \\
Engineering Tests & RAD,SCAT,ALT,SAR & 6 \\
\hline
\end{tabular}

These observations are broken down into several types, as shown in Table I. Note that radar observations are counted per instruction sequence load. In some cases, several adjacent instruction sequence loads occur in close time proximity and really represent one observation opportunity that has been split to address practical sequencing issues.

\section{A. Planned Titan Coverage}

The main science objective of the Cassini RADAR is to map the surface of Titan. The original plan was to cover $25 \%$ or more with SAR imaging, however, this was trimmed back to about $20 \%$ during the integration of the tour sequences. During the mission, SAR imaging has been pushed to cover more area and will likely exceed $25 \%$ by the end of the four-year tour. Fig. 1 shows the planned SAR imaging coverage.

The emphasis of the radar tour designs is on maximizing SAR imaging coverage. The T3 swath shown was extended by pushing SAR to $5000-\mathrm{km}$ altitude at the expense of altimetry time. The T8 design extends the SAR swath with a special pointing design called "pushbroom," which is discussed in more detail later. This option may be used to increase the amount of coverage at the expense of image quality. The planned altimeter tracks are pushed as high as signal-to-noise (SNR) considerations allow, which means about 9000-km altitude. This may be altered if altimetry proves interesting and possible at higher altitudes. Scatterometry scans are positioned to cover new area when possible, and are generally limited to a $60^{\circ}$ incidence angle, although some early scatterometry scans reached incidence angles above $80^{\circ}$. Radiometry scans are also positioned to cover as much area as possible and to provide polarization and incidence angle measurement diversity.

\section{B. Shared Passes and Ride Along Observations}

Limited observing time during Titan flybys has led to many design tradeoffs between instruments and some highly optimized designs. The first flyby (Ta) is one example where optical remote sensing was performed during the inbound portion of the flyby, with a radar scatterometry scan inserted to cover the Huygens probe landing area, followed by atmospheric characterization by the INMS up to the point of closest approach. During the INMS observation, radar SAR data were collected while the spacecraft attitude was compatible with both observation types. Such ride along SAR observations are planned occasionally throughout the tour and also in the extended mission. At the point of closest approach for $\mathrm{Ta}$, RADAR was given primary control of spacecraft attitude, and the outbound sequence includes all of the radar modes. Three
SAR passes (T17, T18, T36) are exclusively ride along SAR, meaning that INMS is the prime instrument while RADAR operates in imaging SAR mode as long as the antenna beams intercept Titan's surface.

\section{Icy Satellite Observations}

The Saturn tour will include many flybys of some of the icy satellites. These bodies are smaller than Titan, and unlike the giant moon, they are not used to control the spacecraft trajectory. Some of these flybys are targeted and come within a few hundred kilometers from the surface. Since these bodies have little to no atmosphere, the optical remote sensing instruments are used around closest approach to take advantage of their higher resolution and spectroscopic capabilities. At longer ranges, the radar instrument is operated to provide complementary unique information that can be used to help decipher the surface properties of these icy satellites.

Since the radar operates at very high ranges of 50000 to $420000 \mathrm{~km}$ during these observations, SAR and altimetry observations are not possible. Instead, disk integrated scatterometry and radiometry data are collected. In some cases, the central beam is about half the apparent angular size of the target body, and a multipoint observation is conducted to look for large-scale variation in surface properties. In other cases, the central beam is larger in angular extent than the target body, and a single stare centered on the target is conducted. Radiometry data are collected during the scatterometer integrations and also during a small raster scan centered on the target body. The raster scan provides on- and off-target measurements to help calibrate the radiometer. The scatterometer integrations usually involve transmitting a single frequency carrier and then recording the Doppler-shifted and spread echoes from the target for five to $20 \mathrm{~min}$. The measured echoes are processed in the frequency domain to provide an echo power spectrum and a low-noise disk-integrated radar cross-sectional measurement. In some cases, a chirped pulse is transmitted to allow for range processing. Chirped pulses are only used when there is sufficient predicted SNR and some ambiguity-free illuminated area.

\section{Saturn Radiometry}

The SOP includes five radiometry observations dedicated to observing Saturn's disk at relatively close ranges. Three of the five were part of a pole to pole global scan conducted during the periapsis of orbit 15 when the spacecraft was near Saturn's equatorial plane. The range to Saturn during these scans varied from about $3 R_{\mathrm{s}}$ to about $7.5 R_{\mathrm{s}}$, where $R_{\mathrm{s}}=60268 \mathrm{~km}$ is the radius of Saturn. These scans covered about $250^{\circ}$ of longitude with resolution varying from 800 to $3000 \mathrm{~km}$. The resulting brightness temperature measurements show much latitudinal and longitudinal structure in the thermal emission of Saturn's atmosphere. As discussed in [1], these data in combination with data from other instruments will be used to explore Saturn's atmospheric structure and motion. The other two Saturn radiometry observations cover the North and South polar regions in February 2008.

\section{E. Rings Radiometry}

The 12 rings observations are designed to derive information about ring particle scattering and thermal properties. Ku-band 


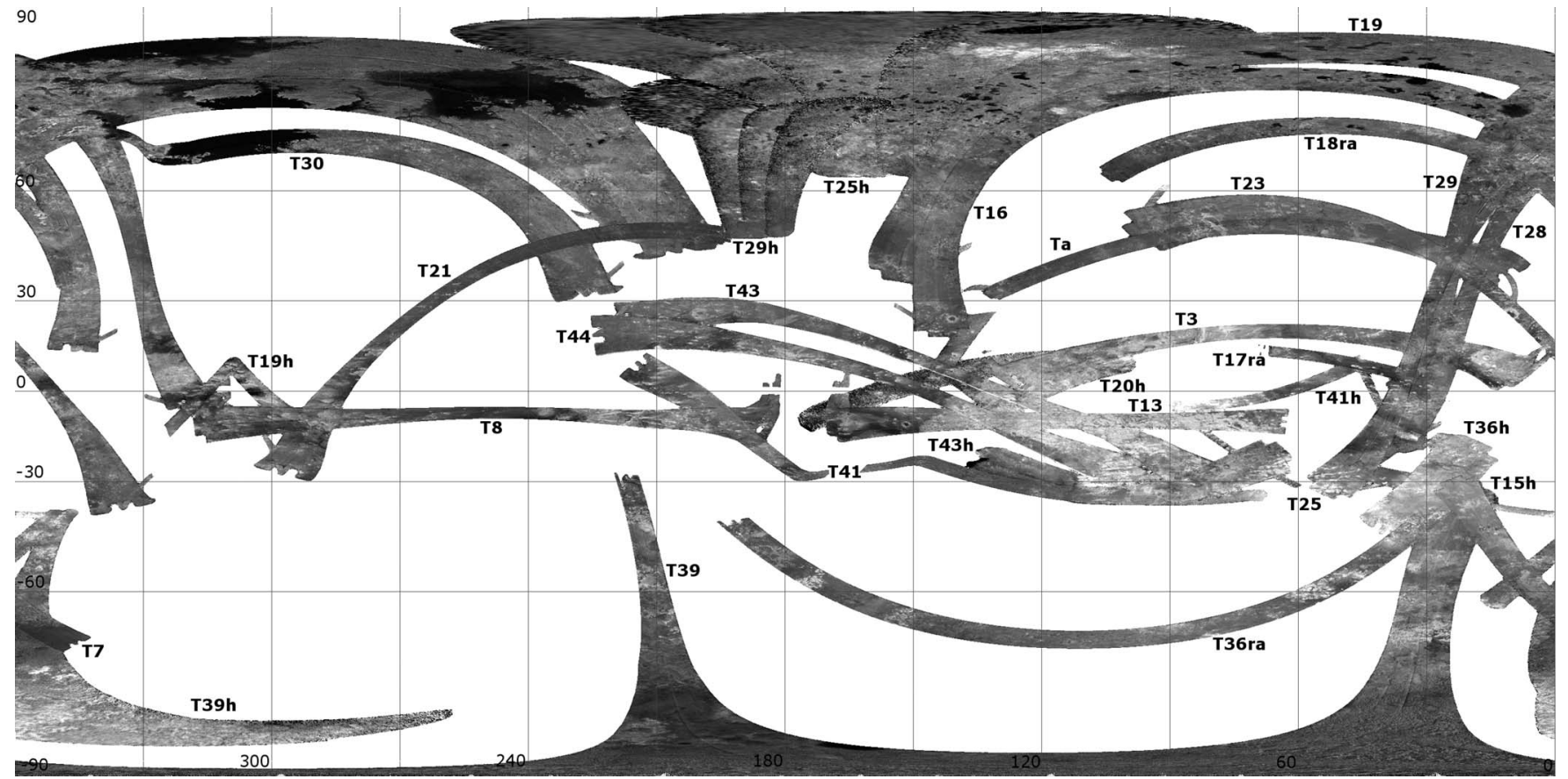

Fig. 1. SAR coverage during the four-year prime mission. West longitudes are used. Each flyby swath is labeled according to the Cassini Project designation given to the corresponding Titan flyby. The prime mission includes 45 Titan flybys designated Ta, Tb, Tc, T3-T44. A suffix of ra indicates a ride-along observation, and a suffix of $\mathrm{h}$ indicates a high-altitude imaging observation. Three flybys (Ta, T21, T39) are half ride-along, but this distinction is not made on the map. The map is produced using a Miller projection.

radiation from the rings consists primarily of emission from Saturn scattered by ring particles into the direction viewed by the antenna beam. Many of the ring scans consist of radial spoke scans which provide data at different scattering angles as a function of radial position. The radiometer data provide more information if two measurements at the same scattering angle and two orthogonal polarizations can be obtained. Since the radar feeds to the high-gain antenna have a fixed linear polarization with the E-field along the spacecraft $X$-axis, changing the observation polarization involves rotating the spacecraft about the $Z$-axis. The spoke scans are then repeated with different spacecraft orientations to provide the desired polarization diversity. Some of the scans are performed on the unlit side to observe changes in emission from the ring particles themselves. These data are then used to interpret the physical and thermal properites of the ring particles at different radial distances.

\section{F. Calibration Observations}

In addition to the science-oriented measurements, there are a number of cosmic source observations and engineering tests that are conducted periodically to provide calibration data. The radiometric cosmic source scans are of radio sources M17, Cassiopeia-A, Taurus-A, and Orion-A. These sources are all bright ground-based radio sources and provide an absolute reference for the radiometer calibration. The engineering tests use Saturn as a reference to measure system performance in all of the operating modes and with various attenuator settings. Saturn is a good absolute calibration reference because it provides a relatively strong emission signal of about $148 \mathrm{~K}$, which fills the main lobe of the central beam. At the same time, the emission from Saturn's atmosphere is probably the simplest to model and the disk-integrated emission is easily observed from
Earth. Distant Titan radiometer and scatterometer observations can also provide reference data for calibration since Titan has also been observed by radar systems on the Earth [2]-[5]. The Huygens probe has provided a temperature measurement on the surface [6], and this may provide the best absolute calibration reference of all.

\section{RADAR SEQUENCE DESIGN}

The high-level design of the Cassini RADAR is described in [1, Secs. 5 and 6]. Elachi et al. [1, Secs. 3 and 4] summarize high-level radar sequence designs and system performance issues. In this section, we will delve more deeply into the design tradeoffs made when putting together the radar observation sequences.

A radar observation consists of two separate but tightly coordinated parts. The first part is the pointing design, which specifies the spacecraft attitude variation during the radar observation. Spacecraft pointing design tools are used to generate pointing commands that track targets and perform raster scans and other tailored pointing sequences. These pointing design tools are used by all of the instrument teams. For the SAR mode, a radar-specific special-purpose program called DLAP_GENERATE was developed to produce a spacecraft attitude profile optimized for SAR imaging performance. DLAP stands for desired look-angle profile.

The second part is the instrument execution block (IEB), which consists of a sequence of instructions executed by the radar flight computer. While executing a radar instruction sequence, data are recorded by the radar flight computer and periodically transferred to the spacecraft computer for later transmission to Earth. The spacecraft has to point its high-gain antenna at the Earth to transmit data, so the entire observation 
sequence executes in the blind. The IEB specifies the operating mode of the radar and all of the necessary pulse parameters including range gate and Doppler offset. The latter two in particular need to be synchronized with the viewing geometry, which depends on the target relative spacecraft position and attitude. A special purpose program called the Radar Mapping and Sequencing Software (RMSS) was developed to semiautomatically generate the radar IEB. The pointing design is generated first by DLAP_GENERATE and other pointing design tools and then input into RMSS along with a set of configuration parameters that describe the detailed design. The following sections describe the pointing and IEB design strategies used for each of the operating modes.

\section{A. SAR Imaging}

SAR coverage of Titan's surface is considered the top priority when it comes to Titan observations, so SAR imaging is pushed as far as possible against the limits imposed by the instrument design, spacecraft resource allocation, and the flyby geometry. SAR imaging by the Cassini RADAR is constrained in practice by the following main factors:

1) round trip time and data buffer size;

2) allocated data volume;

3) number of looks (i.e., spacecraft flyby speed);

4) thermal SNR;

5) ambiguities.

The first three apply mainly to the IEB design, while the last three apply to the pointing design. The number of looks [item 3)] is affected by the pointing design, which sets the speed of motion of the beam footprints on the surface, and by the IEB design, which sets the burst rate.

The SAR pointing and IEB designs are guided by two general and often competing principles. The first is to maximize image area, while the second is to maximize image quality. During SAR imaging, the spacecraft tilts the radar beams left or right of the nadir track; the varying amount of tilt angle is called the look-angle profile. Higher look angles result in higher incidence angles, which means more area illuminated by the beams and better cross-track resolution due to the larger range variation within the illuminated area. However, higher incidence angles also increase the effect of range ambiguities and decrease SNR because of the greater range to the surface. In practice, these competing effects are balanced against each other by computing the cross-track extent as a function of incidence angle and along-track position which satisfies the following two criteria:

1) noise equivalent $\sigma_{0}<-10 \mathrm{~dB}$;

2) signal-to-ambiguity ratio $>15 \mathrm{~dB}$.

The incidence angle that maximizes the usable cross-track extent is selected for each along-track position. Usable crosstrack is defined to be the contiguous cross-track section that satisfies the two criteria above. The resulting profile is fit by an eighth-order polynomial as a function of altitude above the surface. The coefficients of this profile are supplied to the SAR pointing tool DLAP_GENERATE, which uses the profile along with a selected tracking option to produce the spacecraft attitude during the SAR imaging time. Since Titan's radius of $2575 \mathrm{~km}$ is comparable to the ranges encountered during SAR imaging, the spherical nature of the surface is significant, and the look angle needed to produce a particular incidence angle is smaller than the corresponding incidence angle. In principle, the look-angle profile is unique for each flyby altitude, but in practice, a given profile can be applied to a range of altitudes without giving up any noticable imaging performance. Currently, two profiles are used-one optimized for $950-\mathrm{km}$ flybys, and another optimized for $1500-\mathrm{km}$ flybys.

The tracking option determines how the spacecraft rotates to keep the radar beams on the target body, and also how it rotates about the axis of the high-gain antenna. Normally, the tracking option is set to minimize Doppler variation as a function of range within the illuminated areas of each beam. Strictly speaking, the minimum occurs at one point, and the point is chosen to be the center of the central beam. This tracking is called iso-Doppler tracking, and it is used because SAR imaging divides the echo power into range and Doppler pixels. Keeping as much of the Doppler variation orthogonal to the range variation as possible provides relatively square pixels and optimal resolution across the image swath. Iso-Doppler tracking also makes it easier to derive the Doppler centroid from the echo data for each beam, and it reduces the potential for focusing on the wrong Doppler ambiguity.

Another tracking option that has been used is implemented as a variation on the iso-Doppler tracking just described. This option called "pushbroom tracking" adds an azimuth or alongtrack rotation rate over a user specified time interval. The net effect of this is to slow down or speed up the sweep of the five-beam swath along the surface. Usually, the swath sweep is accelerated in the outer portions of the swath to cover more area. The primary tradeoff is a reduction in the number of looks as the same number of bursts are spread over a larger area. The outer portions of many SAR swaths have a surplus of looks in the standard iso-Doppler design, so this can be a good tradeoff. If taken too far, then the usable cross-track extent is reduced because of intruding range ambiguities and diminished SNR as the incidence angle increases away from the optimized profile. This option was first used on the T8 Titan flyby, and will be used judiciously to increase imaging coverage on future Titan flybys. One example is the T25 Titan flyby that occurred on February 22, 2007. Fig. 2 shows the incidence angle profile for the T25 flyby, which used pushbroom tracking on both ends. T25 was a $1000-\mathrm{km}$ flyby and used the low-altitude look profile. The pushbroom tracking caused the incidence angle to increase at the ends before the turns from and to nadir pointing for the adjacent altimetry. The T25 SAR image swath is shown in Fig. 3.

Once the SAR pointing profile is established, then the IEB is designed around it to maximize the resolution and number of looks within limits imposed by the spacecraft and radar instrument. Overall data volume is the ultimate constraint; however, because of the high priority given to SAR imaging, the IEB parameters for SAR are pushed to the limit while the other active modes are throttled back to stay within the allocated data volume. The Cassini RADAR is a burst mode radar, which means a burst of pulses is transmitted and then the echoes are received as one contiguous data stream. The pulse duty cycle is limited to 70\%, and RMSS tries to keep the instrument operating close to this limit during SAR imaging. Around Titan periapases, burst mode operation limits the number of pulses by the round trip time. Thus, the round trip time becomes the longest synthetic aperture time possible, and this sets the 


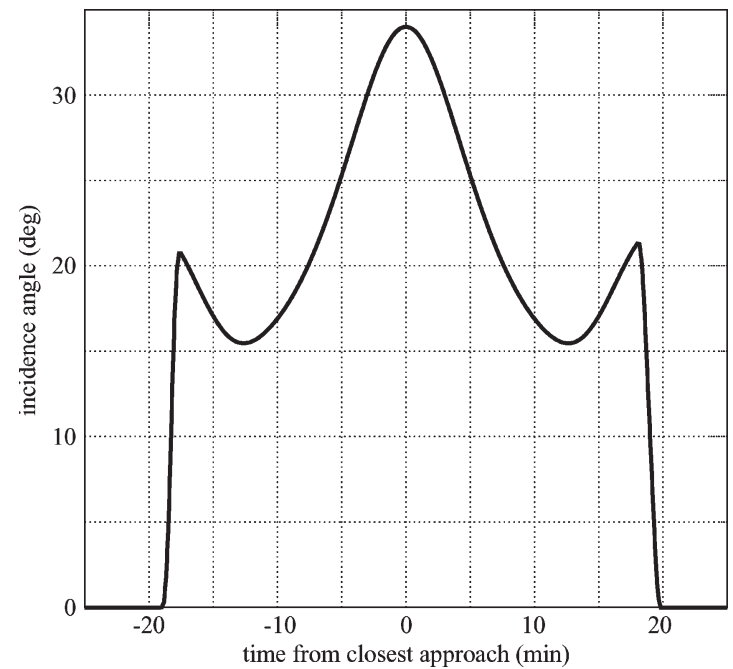

Fig. 2. Incidence angle at center of central beam during the T25 flyby. This shows a typical pushbroomed look-angle profile for a low-altitude flyby where closest approach is below $1200 \mathrm{~km}$.

limit on azimuth resolution. The effective resolution can be calculated from [7, Sec. 1]

$$
\begin{aligned}
\delta R_{\mathrm{g}} & =\frac{c}{2 B_{\mathrm{r}} \sin \theta_{\mathrm{i}}} \\
\delta x & =\frac{\lambda R}{2 \tau_{\mathrm{rw}} v \sin \theta_{\mathrm{v}}}
\end{aligned}
$$

where $\delta R_{\mathrm{g}}$ is the projected range resolution on the surface, $c$ is the speed of light, $B_{\mathrm{r}}$ is the transmitted chirp bandwidth, $\theta_{\mathrm{i}}$ is the incidence angle, $\delta x$ is the azimuth resolution on the surface, $\lambda$ is the transmitted wavelength, $R$ is the slant range, $\tau_{\text {rw }}$ is the length of the receive window, $v$ is the magnitude of the spacecraft velocity relative to the target body, and $\theta_{\mathrm{v}}$ is the angle between the velocity vector and the look direction. RMSS sets the number of pulses to fill the round trip time, and with a 1000-km flyby, the periapases azimuth resolution works out to about $300 \mathrm{~m}$ on the surface. The $2-\mathrm{MHz}$ sample rate is normally used since it produces a surface range resolution of about $500 \mathrm{~m}$, which is comparable to the azimuth resolution. This mode (called Hi-SAR) also keeps the echo buffer smaller than the 32-kB science data buffer for about half of the nominal SAR swath. When the range exceeds about $2400 \mathrm{~km}$, the data buffer is filled, and it is no longer possible to fill the round trip time with pulses. Beyond this range, azimuth resolution will start to deteriorate more rapidly. The synthetic aperture time is fixed, thus fixing the Doppler resolution, while $\theta_{\mathrm{v}}$ and the Doppler spread within the illuminated area diminishes as the spacecraft pulls away from periapsis. At altitudes above $4000 \mathrm{~km}$, azimuth resolution reaches $1 \mathrm{~km}$ on the surface, and in some cases it is better to switch to the 1-MHz sample rate (called Low-SAR), which rebalances range and azimuth resolution and boosts thermal SNR by $3 \mathrm{~dB}$ by cutting the noise bandwidth in half. Fig. 4 shows the variation of imaging resolution during the time around closest approach when SAR imaging is normally performed. The calculations are performed using (1) and (2) for the boresight of beam 3, which is the center of the swath. The rapid steps back and forth at the start of the plot show the effects of switching back and forth between

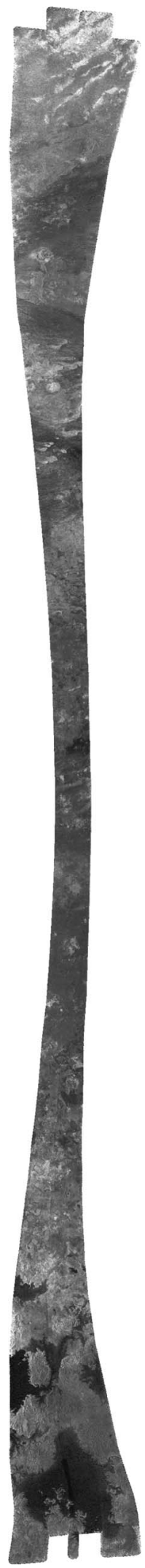

Fig. 3. T25 SAR image swath, projected into an oblique cyclindrical coordinate system aligned with the spacecraft trajectory. This image is averaged down by a factor of ten and mapped into a viewable image using a gamma of 0.4 . The swath is about $6600 \mathrm{~km}$ long. The center of the swath is about $150 \mathrm{~km}$ wide while the ends are about $530 \mathrm{~km}$ wide. Effective resolution varies from $300 \mathrm{~m}$ in the center to about $1 \mathrm{~km}$ at the ends. The spacecraft moved from top to bottom on this figure, and the radar illumination comes from the left side. 


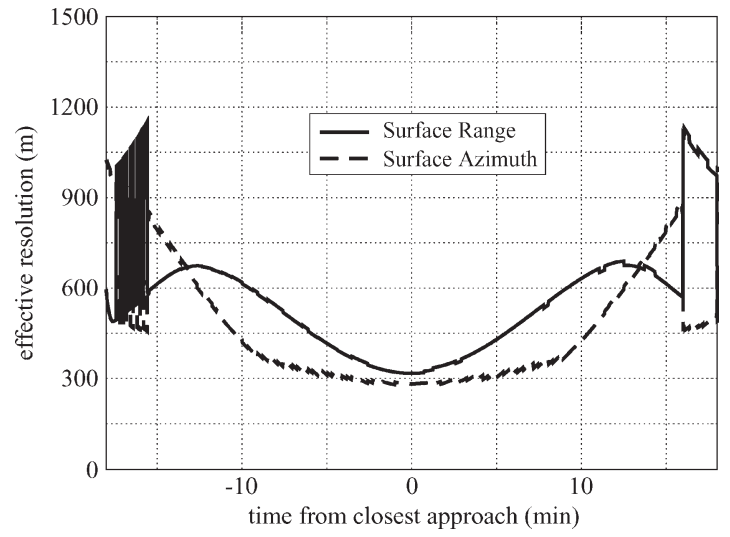

Fig. 4. SAR effective resolution during the T25 flyby. Rapid switching between the Hi-SAR (1-MHz bandwidth) and Low-SAR (0.5-MHz bandwidth) causes the back and forth variation of range and azimuth resolution in the first few minutes. Otherwise, Hi-SAR was used and surface range resolution varies because of varying incidence angle. Azimuth resolution stays relatively constant near closest approach and then deteriorates rapidly once the science data buffer is filled and the synthetic aperture can no longer be extended to compensate for falling Doppler spread away from closest approach.

Low-SAR and Hi-SAR every $12 \mathrm{~s}$. These switches are done to obtain a small increase in image quality because the two modes produce overlapping rectangular pixels with the short side in different directions.

Another important performance limiting constraint is the time needed to recharge the energy storage system (ESS) prior to each transmit burst. The ESS is included in the radar design to even out the load placed on the spacecraft power bus. With the ESS, the radar draws 86 watts while transmitting; however, the transmit bursts are limited to an overall $7 \%$ duty cycle. The ESS also limits the total energy in a burst, but SAR imaging does not approach this limit in practice. The $7 \%$ duty cycle sets a ceiling on the SAR burst rate, limiting the number of looks at periapsis to about three for each of the five beams. The interlude between bursts leaves time for the radar computer to apply an 8- to 2-bit block adaptive quantization (BAQ) to each burst echo buffer, which makes the SAR data collection more efficient and keeps the radar data generation rate below the $365 \mathrm{~kb} / \mathrm{s}$ spacecraft data system limit. The BAQ algorithm used by the radar is very similar to the algorithm used on the Magellan radar mapping mission [8] and is described in the RADAR High-Level Design Document [9].

Away from periapsis, the motion of the beam patterns over the surface slows down while the beam spread gets larger and the number of looks increases dramatically. For a normal isoDoppler profile, the number of looks can reach $30+$ at $4000 \mathrm{~km}$ altitude. RMSS could diminish the burst rate here, but in keeping with the high priority on SAR imaging, we prefer to alter the pointing profile using the pushbroom tracking option described earlier to spread the looks over more area.

Finally, there is one more instrument constraint that has to be considered. The IEB is a list of instructions containing all of the pulse timing parameters along with other settings such as attenuators and radiometer control instructions. Although a new instruction can be issued each second, memory constraints and the radar flight software limit the IEB to 500 instructions. Instructions are primarily needed to track the varying range and Doppler shift. The varying range changes the echo delay time, which is accounted for in the receive window delay setting.
Varying Doppler is compensated by adjusting the chirp start frequency. Both of these settings are updated with each new instruction. To conserve the use of radar instructions, RMSS calculates a valid time for each instruction based on the margins in time and frequency and the derivatives of range and Doppler variation. Instructions are then issued with the spacing set by the valid time. A typical SAR swath uses less than 200 instructions, so no compromise of SAR imaging performance is needed to meet this constraint.

\section{B. High-Altitude SAR Imaging}

SAR imaging was originally only considered for altitudes below $4000 \mathrm{~km}$. There is nothing in the instrument design that precludes imaging at higher altitudes as long as sufficient time and data volume are available. Starting with the T13 Titan flyby, high-altitude imaging segments have been inserted to obtain imaging from altitudes ranging from 11000 to $25000 \mathrm{~km}$. To maintain adequate SNR, only the central high-gain beam is used. At higher altitudes, the central beam spans a wider area, so useful swath widths in the range of 70 to $150 \mathrm{~km}$ can still be obtained. Instead of relying on spacecraft motion to sweep the beam across the surface, these imaging segments pan the central beam across the surface by turning the spacecraft. The position of the beam on the surface is carefully controlled to stay within the area of acceptable viewing geometry, where isorange and iso-Doppler contours cross each other at angles greater than $45^{\circ}$. The spacecraft turn rate controls the sweep rate of the beam on the surface, and this rate is adjusted to provide sufficient looks while covering as much area as possible. Fig. 6 shows results from a high-altitude image segment obtained in the T25 Titan flyby. This image consists of three scan lines positioned to just touch each other along the sweep direction. Scatterometer mode is used to obtain reasonably balanced range and azimuth resolution around $2.5 \mathrm{~km}$, as shown in Fig. 5. The primary constraints on resolution are the limited Doppler spread within the beam footprint and the 32-kB science data buffer, which limits the size of the synthetic aperture. The central beam has enough gain to yield a noise equivalent backscattering cross section of about $-10 \mathrm{~dB}$. The area imaged in Fig. 6 lies near the north pole and many small lakes are evident.

\section{Altimeter Sequence Design}

Altimeter segments occur immediately before and after a SAR swath. They are nadir pointed and cover altitudes between 4000 and $10000 \mathrm{~km}$. The pulse repetition rate is fixed at $5 \mathrm{kHz}$ to allow for potential Doppler sharpening. The highest bandwidth setting is used with a nominal chirp bandwidth of $4.25 \mathrm{MHz}$, yielding a range resolution of $35 \mathrm{~m}$. The $0.37^{\circ}$ beamwidth of the central beam used for altimetry gives a beam limited footprint of 26 to $65 \mathrm{~km}$. The pulse duty cycle is $70 \%$, and the $0.14-\mathrm{ms}$ transmitted pulses yield a pulse limited footprint of $21 \mathrm{~km}$. To maximize the quality of each burst, the number of received pulses is set to 15 , which almost fills the echo data buffer. To increase the valid time and conserve on instructions, the number of transmitted pulses is set to 21 to allow for 6 pulses worth of range walk before a new instruction is needed. Unlike SAR, altimetry is dominated by range variation because the spacecraft is rapidly approaching or 


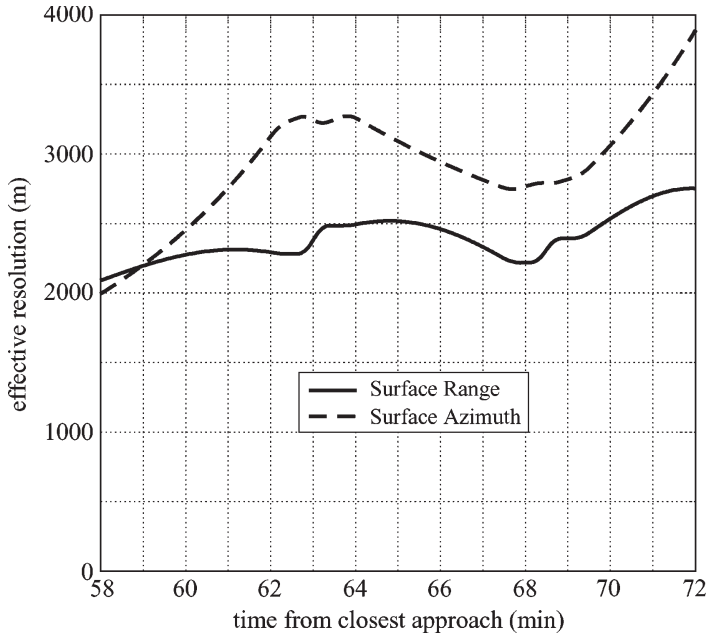

Fig. 5. SAR effective resolution during the T25 north polar high-altitude imaging segment. The high range of around $18000 \mathrm{~km}$ required the scatterometer mode bandwidth $(125 \mathrm{kHz})$ and beam 3 only operation to obtain adequate SNR and the best possible azimuth resolution. Incidence angles lie mostly between $35^{\circ}$ and $40^{\circ}$ during this segment.

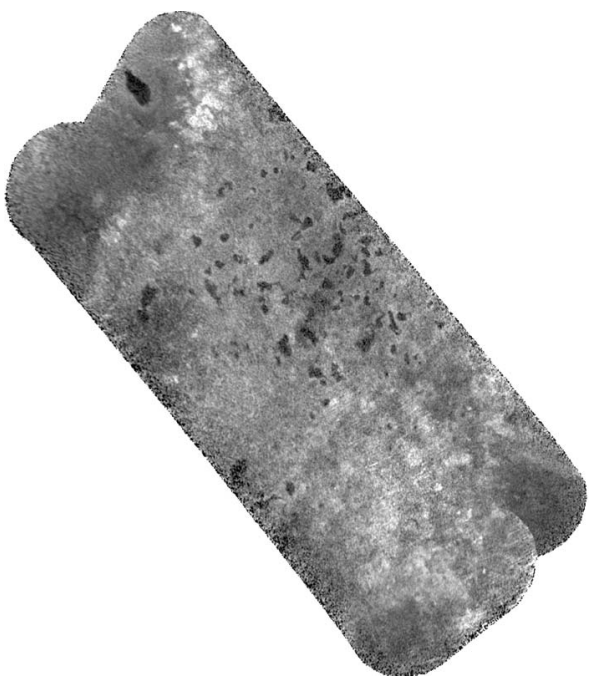

Fig. 6. T25 high-altitude SAR image segment, projected into an oblique cyclindrical coordinate system aligned with the spacecraft trajectory. This image has an effective resolution around $2.5 \mathrm{~km}$ and is mapped into a viewable image using a gamma of 0.4 . The rectangular image area is about $550 \mathrm{~km}$ by $1300 \mathrm{~km}$ in size. The image is formed from three scans of the central beam, and the radar illumination comes from the right on this figure.

receding from Titan when this mode is used. One or two special bursts are inserted with only nine transmitted pulses to allow the leading pulse to be clearly identified. The burst rate determines the data generation rate, and this is limited by available data volume. Nominally, the altimeter operates at $30 \mathrm{~kb} / \mathrm{s}$, just like scatterometry. If more data volume is available, then this rate is increased to provide more burst overlap and potentially better quality altitude results. SNR is generally high, so the altimetry data are compressed using an 8- to 4-bit BAQ.

Range spread within the beam varies from $75 \mathrm{~m}$ to over $200 \mathrm{~m}$ in the normal altimeter segments, and this leads to echo waveforms that spread out over several range bins. Moreover, because the motion of the beam across the surface is slower at higher altitudes (around $2 \mathrm{~km} / \mathrm{s}$ during altimeter segments), the altimeter tracks only cover a few hundred kilometers of track length. These limitations of the nominal altimeter segments make the interpretation of the altimeter data more challenging. To aid the interpretation of altimeter data, these deficiencies were eliminated in the T30 flyby by sacrificing the inbound SAR and instead collecting a very long altimeter data set. The central beam was nadir pointed for the inbound half of the flyby. The resulting altimeter track overlapped with the previous T28 SAR swath, which was positioned to allow this unusual overlap opportunity. The same pulse parameters were used as in regular altimetry collections, but the data rate was pushed up to the limit allowed by the BAQ algorithm on the flight computer. This shortened the burst period and allowed for more looks and Doppler sharpening. The resulting altimeter data set provided a unique high-resolution look at the surface topography because the beam extent at closest approach was only $6 \mathrm{~km}$, and the range spread within the beam due to the beamwidth was about $7 \mathrm{~m}$, which is much less than the range resolution. Long altimeter tracks may be repeated in the extended mission to acquire more high-resolution topographic data in areas of special interest.

\section{Scatterometer Sequence Design}

Scatterometry uses the lowest bandwidth setting with a sample rate of $250 \mathrm{kHz}$ and a nominal chirp bandwidth of $106 \mathrm{kHz}$. The low bandwidth and real aperture processing allow a reliable backscattering measurement to be made at very high ranges.

1) Titan Scatterometry: During Titan flybys, scatterometry data are collected just outside of the altimeter segments. Normally, scatterometry is used during a raster scan that lasts about $45 \mathrm{~min}$ and ranges from $10000-\mathrm{km}$ altitude up to about $30000-\mathrm{km}$ altitude. In some cases, the spacecraft has to transition from thruster control to momentum wheel control, and this can cut the time available for scatterometry by $20 \mathrm{~min}$. The raster scan designs take into account the desire to cover new area and the desire, where possible, to collect data at different polarizations and incidence angles for a given position on the surface. Such measurement diversity coupled with the passive radiometry collected at the same time can help to constrain models of the surface properties [10]. Scan lines are kept on the surface except for the first one, which goes offtarget to provide cold space reference measurements for the coincident radiometry. Unlike altimetry, Titan scatterometry is set to transmit eight pulses and receive 14 pulse repetition intervals. The echo time pad serves two purposes. First, it increases the instruction valid time by allowing the echoes to walk through the longer echo buffer. This is very important for scatterometry because the rapid scan movements make it the biggest consumer of radar instructions. Second, the noiseonly time in the echo buffer provides a noise-only measurement that is used as part of the calibration process described in more detail later. At high incidence angles, the range spread within the beam footprint exceeds the time margin, and this needs to be accounted for in processing. The pulse repetition rate is set to $1.2 \mathrm{kHz}$ for scatterometry, which covers the Doppler spread of the central beam in case Doppler processing is desired. The data rate is nominally $30 \mathrm{~kb} / \mathrm{s}$, but this can be adjusted to stay within the allocated data volume limit.

2) Compressed Titan Scatterometry: In a radar pass that includes SAR and altimetry, there are not enough radar 
instructions or data volume to carry scatterometry out to higher ranges than $30000 \mathrm{~km}$, even though there is enough SNR to do so. Occasionally, the Cassini RADAR is allocated a radiometer-only Titan pass with limited data volume. In these cases, a special compressed scatterometer mode can be employed to acquire backscatter measurements during the radiometry scans. This mode incoherently sums the magnitude of the echo data into one pulse repetition interval. With the pulse parameters set to fill the echo data buffer, performance is improved by transmitting 90 pulses, and a compression of 45 to 1 can be achieved. Since magnitudes are summed, the noise-only data have to be scaled during processing to recover a power value. The data rate in this mode is reduced to about $4 \mathrm{~kb} / \mathrm{s}$ which is suitable for piggy-backing onto a radiometer only segment.

3) Distant Icy Satellite Scatterometry: During Titan scatterometry, each pulse echo has enough SNR to produce a usable backscatter measurement. Icy satellite scatterometry, however, occurs at much higher ranges of 50000 to $400000 \mathrm{~km}$, and the individual echo signals are weaker than the power level of the noise floor. To compensate for the low SNR, many echoes have to be summed together until the echo power greatly exceeds the level of fluctuation in the noise power. Assuming that we have a separate independent estimate of the noise power, the normalized variance $K_{\mathrm{pc}}^{2}$ for a real aperture backscatter measurement is given by [11]

$$
K_{\mathrm{pc}}^{2}=\frac{1}{N \tau_{\mathrm{p}} B}\left(1+\frac{2}{\mathrm{SNR}}+\frac{1}{\mathrm{SNR}^{2}}\right)
$$

where $N$ is the number of pulses averaged together, $\tau_{\mathrm{p}}$ is the pulsewidth, and $B$ is the measurement bandwidth. Taking a square root gives $K_{\mathrm{pc}}$, which is the normalized standard deviation of the backscatter measurement. Normalization here means that the standard deviation of the measurement has been divided by the expected value of the measurement. When SNR is high, the first term dominates and larger chirp bandwidths provide better results because they provide more effective looks that are averaged together to reduce the measurement variance. When SNR is very low, the third term dominates, and measurement bandwidth should be minimized to reduce the effect of noise variance in the measurement. Regular Titan scatterometry falls in between these two extremes, and the nominal 106-kHz chirp bandwidth minimizes the backscatter measurement variance in the 10000 - to $30000-\mathrm{km}$ range.

Icy satellite scatterometry observations usually fall in the very low-SNR regime. Therefore, a tone transmission is used to minimize the measurement bandwidth. Even though the transmitted pulses are very narrow bandwidth tones, the received echo power is spread out in the frequency domain by Doppler variation, which arises from a combination of the relative motion of the spacecraft and the rotation of the target body. For the icy satellites, the Doppler variation ranges from a few hundred hertz up to about $4 \mathrm{kHz}$. The pulsed nature of the transmit events also introduces grating lobes spaced at the pulse repetition frequency (PRF). This causes the echo power spectrum to be repeated at intervals of the PRF. To make processing easier, the PRF is set to a frequency higher than the predicted Doppler spread of the target body so that the central spectral peak is separated from the grating lobes, which shows the natural variation of the echo power over the target body.

The echoes from an icy satellite are sometimes predicted to be strong enough to support limited range and Doppler processing. In these cases, a segment of chirped data is also included when the central beam is centered on the target body. Only centered stares are used because of severe range ambiguity restrictions.

Some icy satellite observations also include receive only segments on and off of the target body. These data are used to calibrate system gain and temperature as discussed later in this paper. To reduce uncertainty due to attenuator variations, all of the icy satellite observations use the same 8.4-dB attenuator setting. This attenuator setting is also used by most of the Titan scatterometry data collections.

\section{E. Radiometer Sequence Designs}

The Cassini RADAR is designed so that radiometer data are always collected regardless of the operating mode. Each radar burst ends with three radiometer measurements. The internal matched load and noise diode are sampled once each, and then the antenna temperature is integrated until the burst period time is used up. During active mode observations, the radiometer antenna integration time varies according to the needs of the active mode data collection. There is always at least one integration window (about $35 \mathrm{~ms}$ ) in each burst. During radiometeronly observations, the antenna integration fills most of the burst period, which is usually set to $1 \mathrm{~s}$. A 1-s radiometer integration provides measurement variance better than $0.1 \mathrm{~K}$ while limiting beam motion to less than $30 \%$ of a beamwidth for the fastest moving pointing designs.

Most of the design work for radiometry sequences goes into the spacecraft pointing design. For Titan flybys, the altitude range from $30000 \mathrm{~km}$ up to $100000 \mathrm{~km}$ is considered radiometer-only because of diminishing SNR in the active modes. In some cases, there is enough data volume available to collect compressed scatterometry during this altitude range, but the pointing design is still dictated by the needs of the radiometer observation. Normally, two overlapping raster scans are executed with the edges lying at least three beamwidths beyond the limb. The off target edges allow for the removal of gain drift during the scans. In between the two scans, the spacecraft rolls about the $Z$-axis by $90^{\circ}$ so that the second scan sees the same surface with a different measurement polarization. The overlap is not perfect because one scan is closer and has higher resolution, but the polarization diversity is still very valuable in characterizing surface properties. The raster scans naturally cover a wide range of incidence angles, and this variation in combination with the polarization variation can be used to identify the Brewster angle and the effective dielectric constant of the surface.

\section{RADIOMETRIC CALIBRATION}

The Cassini RADAR can be separated into a set of active modes that differ in bandwidth and a passive radiometry mode. The active and passive mode receiver systems share some key hardware elements, as shown in Fig. 7. The high-gain antenna, feedhorns, waveguides, front-end switch matrix, and low-noise 


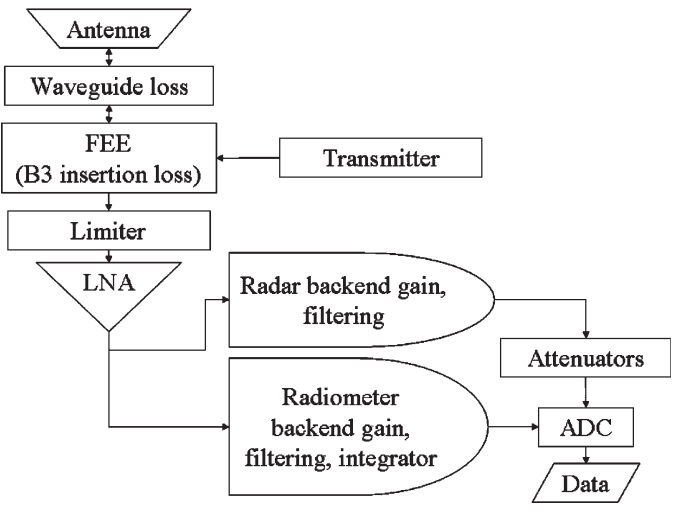

Fig. 7. Simplified RF schematic. Passive and active systems share the same antenna, front-end switch network and LNA. The radiometer receive path then splits off through a relatively wide filter $(125 \mathrm{MHz})$ and an analog integrator/detector. The radar receive path passes through successive filters, attenuators, and mix-down stages that are synchronized with the transmitted chirp. Four different bandwidths can be selected. The final conversion to digital data is performed by an 8-bit analog-to-digital converter.

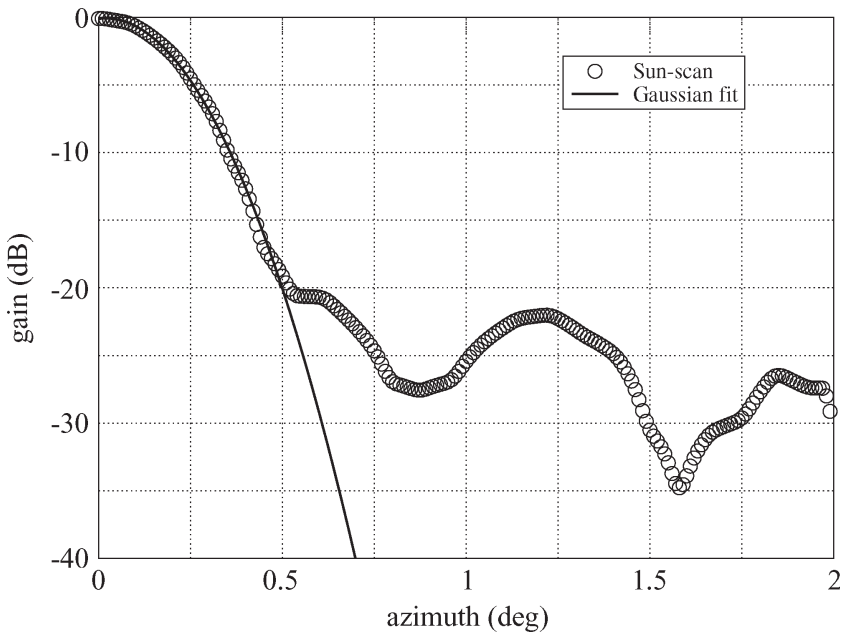

Fig. 8. Azimuth cut of the beam 3 normalized antenna pattern. Measurements from a sun scan and a Gaussian fit to the main lobe are shown.

amplifier (LNA) are all used by both the radar receiver and the radiometer receiver. The radar receiver diverges after the LNA and passes through a series of attenuators and filters that produce the four active mode bandwidths and 8-bit digitized data. The radiometer receiver uses a much wider bandwidth $(125 \mathrm{MHz})$ and a square-law detector and analog integrator to produce 20-bit digitized data. An internal matched load and noise diode source can also be switched in just before the LNA to provide reference measurements.

\section{A. Antenna Gain Patterns}

Knowledge of the five beam patterns is important to both passive and active calibration. The central beam (beam 3 ) is of particular importance since all of the radar modes use it. During the seven-year cruise to Saturn, several radiometer scans of the sun were performed to collect data on the antenna beam patterns. Fig. 8 shows a 2-D cut in the beam 3 normalized antenna pattern obtained from sun scan data. Also shown is a Gaussian least squares fit to the main lobe. The measured
TABLE II

Antenna Central Beam Parameters

\begin{tabular}{|c|c|}
\hline$\theta_{\mathrm{bw}}$ & $0.373 \mathrm{deg}$. \\
$\Omega_{p}$ & $7.55 \mathrm{e}-5 \mathrm{rad}^{2}$ \\
$\Omega_{M}$ & $4.80 \mathrm{e}-5 \mathrm{rad}^{2}$ \\
$\eta_{M}$ & 0.64 \\
$\delta \theta_{a}$ & $0.02 \mathrm{deg}$. \\
$\delta \theta_{e}$ & $-0.03 \mathrm{deg}$. \\
$G_{p}$ & $50.7 \mathrm{~dB}$ \\
$D_{0}=4 \pi / \Omega_{p}$ & $52.2 \mathrm{~dB}$ \\
\hline
\end{tabular}

pattern extends out to plus and minus $2^{\circ}$ from the peak, which covers several of the near sidelobes. Integrating the available measured pattern gives a lower bound for the pattern solid angle

$$
\Omega_{\mathrm{p}}^{\prime}=\iint_{\text {meas. pattern }} g(\Omega) d \Omega
$$

where $g(\Omega)$ is the measured gain pattern in the direction $\Omega$ normalized to unity at the peak. Calibrating the radiometer and radar receiver using known reference sources requires knowledge of the full pattern solid angle given by

$$
\Omega_{\mathrm{p}}=\iint_{4 \pi} g(\Omega) d \Omega .
$$

Since the antenna uses a Cassegrain feed system, there are supporting struts for the secondary reflector that cause some scattering, thereby raising the floor of the gain pattern at larger angles from the boresight. Both the secondary and primary reflectors are underilluminated, so spill-over sidelobes are not a significant issue. When the spacecraft is close to a large target such as during the SAR imaging swaths, the radiometry data are affected by the extended gain pattern which can be estimated using a bootstrap procedure described in [12]. These data show that about $35 \%$ of the power radiated by the antenna using beam 3 goes into the extended pattern outside of the measured pattern. Thus, $\Omega_{\mathrm{p}} \approx 1.35 \Omega_{\mathrm{p}}^{\prime}$. Integrating the Gaussian fit shown in Fig. 8 yields the main lobe solid angle $\Omega_{\mathrm{M}}$ and the main lobe efficiency $\eta_{\mathrm{M}}$. The least squares fit also supplies the half-power beamwidth $\theta_{\mathrm{bw}}$ and the azimuth and elevation offsets $\left(\delta \theta_{\mathrm{a}}, \delta \theta_{\mathrm{e}}\right)$ of the electrical boresight from the antenna coordinate frame. Table II summarizes these measurements for the central beam along with a prelaunch measurement of the peak gain. The other four offset beams are used when we want to collect SAR imaging data over a wider swath. Their patterns were also measured with sun scans and used by the SAR processor during image formation.

As expected, the directivity is larger than the peak gain. The measured peak gain includes losses in the antenna subsystem, which were measured on an engineering model to be $2.46 \mathrm{~dB}$. Thus, the measured gain and directivity appear to be reasonably consistent. Since the antenna subsystem losses are incorporated in the measured peak gain value, these losses do not need to be accounted for separately in the radar equation when calibrating the received power. Potentially, these losses could become a factor in the radiometer calibration model, particularly if some 


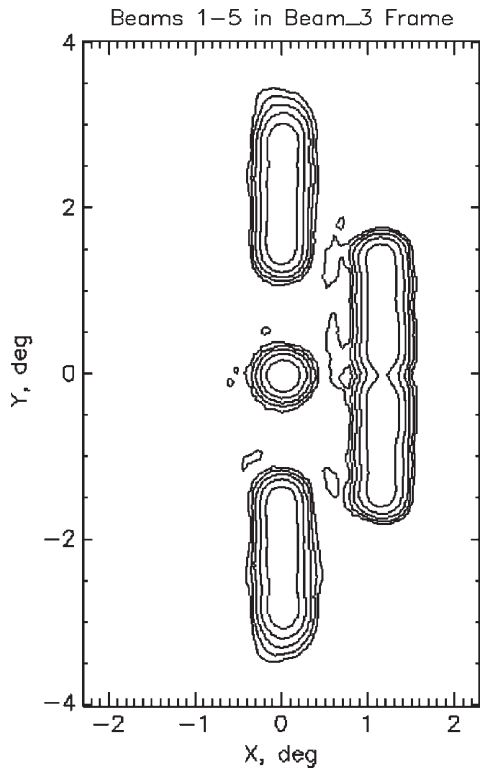

Fig. 9. Contour plot of the five beam patterns using data from the $\mathrm{C} 27$ sun scan obtained during the cruise out to Saturn. The five beams are all produced from a 4-m parabolic dish with feedhorns in the base (Cassegrain feed system). The small central beam has about $6.5 \mathrm{~dB}$ higher gain than the offset outer beams. The middle outer beams are offset in azimuth by $1.2^{\circ}$ to physically accommodate the feedhorns in the base. The five beams overlap in elevation to provide a larger image swath.

component is due to ohmic loss from part of the antenna subsystem.

The other four beams are only used for SAR imaging and associated radiometry obtained at the same time. A switch matrix routes the power from one of the five feedhorns at the base of the parabolic reflector to the LNA. There are some path differences between the beams; however, these loss differences are absorbed by the measured beam patterns, which are all referenced to the peak gain of the central beam. Fig. 9 shows a contour plot of the five beams together. The outer beams are elongated in the elevation ( $Y$-axis) direction to provide more SAR image width. The two midbeams are offset in the azimuth ( $X$-axis) direction by about $1.2^{\circ}$ to physically accommodate the feedhorns. All five feedhorns are polarized with the electric field in the $X$-axis direction. Additional information about the Cassini antenna subsystem can be found in [13, Sec. 5.3].

\section{B. Radiometer Receiver Gain and Noise Temperature}

All of the elements of the radiometer receiver system can be lumped together into an equivalent noise-free system with an overall effective receiver noise temperature $\left(T_{\mathrm{r}}\right)$ and receiver gain $(G)$, both referenced at the antenna port. Each burst includes a radiometer measurement from the antenna as well as from the internal matched load and noise diode. After normalizing out the integration time, the antenna measurement $\left(V_{\mathrm{n}}\right)$ is given by

$$
V_{\mathrm{n}}=G\left(T_{\mathrm{r}}+T_{\mathrm{a}}\right)
$$

where $T_{\mathrm{a}}$ is the antenna temperature. Combining two measurements of different but known sources gives a solution for the two unknowns $G$ and $T_{\text {r }}$

$$
\begin{aligned}
G & =\frac{V_{\mathrm{n} 1}-V_{\mathrm{n} 2}}{T_{\mathrm{a} 1}-T_{\mathrm{a} 2}} \\
T_{\mathrm{r}} & =\frac{V_{\mathrm{n} 1} T_{\mathrm{a} 2}-V_{\mathrm{n} 2} T_{\mathrm{a} 1}}{V_{\mathrm{n} 2}-V_{\mathrm{n} 1}}
\end{aligned}
$$

where the subscripts 1 and 2 refer to the two known sources. The cosmic microwave background (CMB) usually serves as one reference level, while various calibration targets, including Saturn, the satellites of Saturn, Jupiter, the sun, and four galactic microwave sources, are observed periodically to supply the other reference level. The internal noise diode also supplies a potential calibration reference level, although the long-term stability of noise diodes can be problematic as discussed in [14]. In practice, the radiometer receiver has proven to be exceptionally stable and the noise diode data has not been needed to calibrate the radiometer data.

Antenna temperature $T_{\mathrm{a}}$ is defined by (see $[15$, Secs. 4 and 5])

$$
T_{\mathrm{a}}(\Omega)=\frac{\iint_{4 \pi} T_{\mathrm{b}}\left(\Omega^{\prime}\right) g\left(\Omega^{\prime}, \Omega\right) d \Omega^{\prime}}{\Omega_{\mathrm{p}}}
$$

where $\Omega$ refers to the direction the antenna is pointed toward, $g\left(\Omega^{\prime}, \Omega\right)$ is the antenna power gain in the direction $\Omega^{\prime}$ when the antenna is pointed in the direction $\Omega$, and $T_{\mathrm{b}}\left(\Omega^{\prime}\right)$ is the apparent brightness temperature of the area viewed by the antenna toward direction $\Omega^{\prime}$. For the distant Titan and icy satellite observations, the target may not fill the beam main lobe. In these cases, the brightness temperature can be split into two discrete parts

$$
T_{\mathrm{b}}(\Omega)= \begin{cases}T_{\mathrm{as}}, & \Omega \text { off the target disk } \\ T_{\text {target }}, & \Omega \text { on the target disk }\end{cases}
$$

where $T_{\mathrm{as}}=2.7 \mathrm{~K}$ is the microwave background radiometric temperature at $2.2 \mathrm{~cm}$, and $T_{\text {target }}$ is the target effective disk brightness temperature. Note that the target blocks the microwave background when it is in view. Combining equations gives

$$
\begin{aligned}
& T_{\mathrm{a}}(\Omega) \\
& \qquad=\frac{\iint_{4 \pi} g\left(\Omega^{\prime}, \Omega\right) T_{\mathrm{as}} d \Omega^{\prime}+\iint_{\text {disk }}\left(T_{\text {target }}-T_{\mathrm{as}}\right) g\left(\Omega^{\prime}, \Omega\right) d \Omega}{\Omega_{\mathrm{p}}}
\end{aligned}
$$

where the disk integral is taken over the target disk. $T_{\text {as }}$ and $T_{\text {target }}$ are constant so they can be pulled out of the integrals

$$
T_{\mathrm{a}}(\Omega)=T_{\mathrm{as}}+\left(T_{\text {target }}-T_{\mathrm{as}}\right) \frac{\iint_{\text {disk }} g\left(\Omega^{\prime}, \Omega\right) d \Omega^{\prime}}{\Omega_{\mathrm{p}}} .
$$

When the target is outside the beam (at least three beamwidths away from the boresight), then the antenna main lobe sees only the cosmic background, and $T_{\mathrm{a}} \approx T_{\text {as }}$.

Before we can apply these equations, the effect of physical temperature variation of the instrument itself needs to be taken into account. Fig. 10 shows the normalized counts from the antenna during five engineering tests as a function of the LNA 


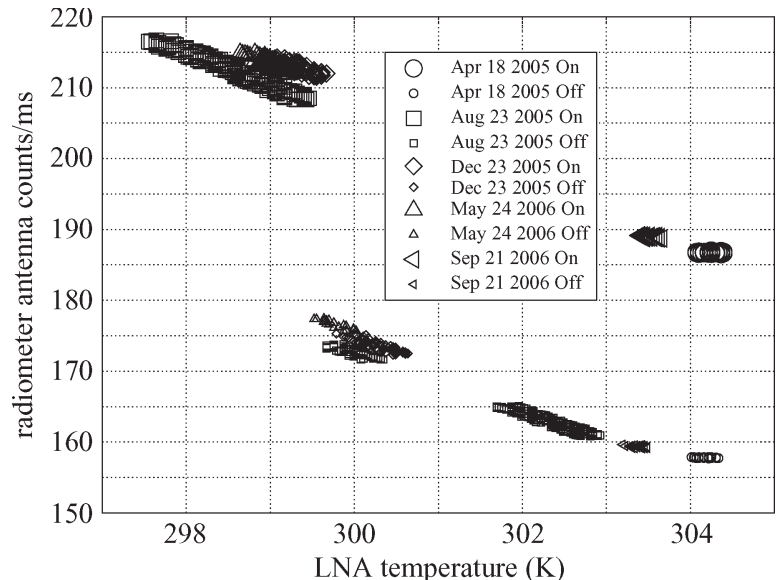

Fig. 10. Normalized antenna counts from the radiometer measured while observing the CMB and Saturn and while the instrument warmed up after turn on. Instrument warmup causes about $1 \mathrm{~dB}$ of drop in gain and takes about $3 \mathrm{~h}$.

TABLE III

RAdiometer SATURn Measurement Summary

\begin{tabular}{|c|c|c|c|c|c|}
\hline Date & $\begin{array}{c}V_{n 1} \\
\text { counts/s }\end{array}$ & $\begin{array}{c}V_{n 2} \\
\text { counts/s }\end{array}$ & $\begin{array}{c}G \\
\text { counts/(s K) }\end{array}$ & $\begin{array}{c}T_{r} \\
\mathrm{~K}\end{array}$ & $\begin{array}{c}d T_{r} \\
\mathrm{~K}\end{array}$ \\
\hline Apr 18, 2005 & 189869 & 160119 & 281 & 567 & 8 \\
Aug 23, 2005 & 192345 & 161282 & 293 & 548 & 8 \\
Dec 23, 2005 & 196060 & 163023 & 308 & 526 & 8 \\
May 24, 2006 & 194547 & 163475 & 302 & 538 & 8 \\
Sep 21, 2006 & 191712 & 160962 & 287 & 558 & 8 \\
\hline
\end{tabular}

physical temperature. After system turn-on, the temperature of the internal components rises almost $15 \mathrm{~K}$. The rise in temperature causes a 30\% drop in the antenna counts due mostly to reduced gain. The dependence of antenna counts on LNA temperature is nearly linear, so a linear least-squares fit is computed and used to adjust the on- and off-target measurements to a common LNA temperature. Table III summarizes the radiometer performance results from five engineering tests that observed Saturn and the CMB. The uncertainties $(d T r)$ shown are $1 \sigma$ errors due to statistical uncertainty in each measurement. Only the April 18, 2005 and September 21, 2006 observations were fully warmed up, and the gain and receiver temperatures in Table III show about $10 \%$ variation during warmup. From data taken during cruise, we find that the warmed up radiometer gain and receiver temperature are stable to within about $2 \%$. If we take the April 18, 2005 result for receiver temperature and compute the gain from just the CMB observations, then we find the gain (see Fig. 11) is consistent to within 2\%. The variation is likely coming from unmodeled systematic effects such as small temperature changes in different parts of the system, and possibly from contamination by scattering from the rings that were in the field of view for some of these data takes. More system tests are scheduled later in the tour to address these issues and to continue assessing the performance of the system.

\section{Radar Receiver Gain and Noise Temperature}

In a nominal "textbook" receiver, the front-end LNA would be expected to dominate $T_{\mathrm{r}}$ (see [15, Secs. 6 and 5]). The radiometer mode could then be used to measure $T_{\mathrm{r}}$, which would

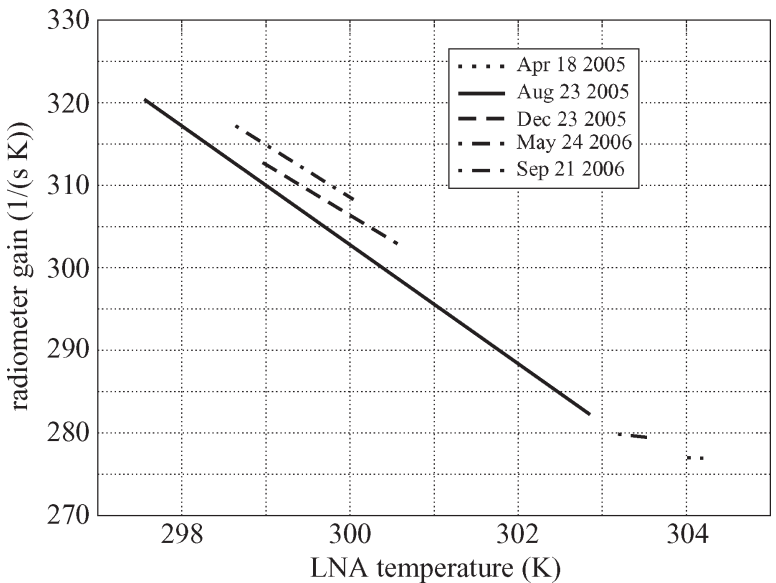

Fig. 11. Radiometer gain assuming $T_{\mathrm{r}}=567 \mathrm{~K}$ and using $\mathrm{CMB}$ data from four engineering tests. System gain decreases as LNA temperature increases during warmup after initial power on. Variation between tests is within $2 \%$.

then provide the baseline calibration for the active modes. However, in-flight test data indicate that the Cassini RADAR active mode receiver is influenced by narrow bandwidth noise or contamination signals. The precise origin of this noise is unknown, but it is possible to measure and account for the extra noise power. Total noise power $P_{\mathrm{n}}$ referenced at the antenna port is the sum of front-end noise power $P_{\mathrm{r} 0}$ and some narrowband contamination power $P_{\mathrm{rf}}$, also referenced at the antenna port. The extra noise power is converted to equivalent noise temperature and added to the overall receiver noise temperature

$$
\begin{aligned}
T_{\mathrm{r}} & =T_{\mathrm{r} 0}+T_{\mathrm{rf}} \\
P_{\mathrm{rf}} & =k T_{\mathrm{rf}} B \\
P_{\mathrm{r} 0} & =k T_{\mathrm{r} 0} B
\end{aligned}
$$

where $T_{\mathrm{r} 0}$ is the front-end receiver noise temperature as measured by the radiometer, $T_{\mathrm{rf}}$ is the extra narrowband noise power expressed as an equivalent temperature, $k$ is Boltzmann's constant, and $B$ is the active mode noise bandwidth selected. $T_{\mathrm{r} 0}$ matches the radiometer receiver temperature and is independent of bandwidth and attenuator setting, while $T_{\mathrm{rf}}$ and therefore $T_{\mathrm{r}}$ vary with attenuator setting and bandwidth. To properly account for the extra noise power, it is necessary to calibrate each attenuator setting used for each active mode.

After passing through the radar receiver, the received noise power is delivered to the analog-to-digital converter, which converts it to 8-bit data with a variance $V_{\mathrm{n}}$ proportional to the noise power

$$
V_{\mathrm{n}}=\frac{C}{\mathrm{a}}\left(P_{\mathrm{r} 0}+P_{\mathrm{a}}+P_{\mathrm{rf}}\right)
$$

where $C$ is the receiver gain incorporating the analog-to-digital conversion constant between watts and data counts, the limiter loss, the LNA gain, and the backend gain, but excluding the attenuator loss factor $L_{\mathrm{a}} . P_{\mathrm{a}}$ is the power from the antenna, which depends on the target being observed. If we define the combined receiver gain as

$$
G=\frac{C k B}{L_{\mathrm{a}}}
$$


TABLE IV

April 18, 2005 Radar Receiver Saturn Measurement Summary

\begin{tabular}{|c|c|c|c|c|c|c|c|c|c|}
\hline $\begin{array}{c}B \\
(\mathrm{kHz})\end{array}$ & $\begin{array}{c}L_{a} \\
(\mathrm{~dB})\end{array}$ & $\begin{array}{c}\text { Saturn } \\
\operatorname{Var}\end{array}$ & $\begin{array}{c}\mathrm{CMB} \\
\operatorname{Var}\end{array}$ & $\begin{array}{c}C \\
(1 / \mathrm{W})\end{array}$ & $\begin{array}{c}d C \\
(\mathrm{~dB})\end{array}$ & $\begin{array}{c}T_{r} \\
(\mathrm{~K})\end{array}$ & $\begin{array}{c}d T_{r} \\
(\mathrm{~K})\end{array}$ & $\begin{array}{c}T_{r 0} \\
(\mathrm{~K})\end{array}$ & $\begin{array}{c}T_{r f} \\
(\mathrm{~K})\end{array}$ \\
\hline 117 & 14.2 & 119.2 & 107.3 & $1.84 \mathrm{e} 18$ & 0.02 & 950 & 14 & 567 & 383 \\
468 & 14.2 & 355.4 & 305.8 & $1.92 \mathrm{e} 18$ & 0.01 & 649 & 9 & 567 & 82 \\
935 & 14.2 & 502.6 & 428.7 & $1.43 \mathrm{e} 18$ & 0.02 & 611 & 9 & 567 & 44 \\
4675 & 14.2 & 2371.9 & 1998.9 & $1.44 \mathrm{e} 18$ & 0.01 & 565 & 11 & 567 & -3 \\
125000 & 0 & 189869 & 160119 & $1.63 \mathrm{e} 17$ & 0.0005 & 567 & 8 & 567 & 0 \\
\hline
\end{tabular}

TABLE V

September 21, 2006 Radar Receiver Saturn Measurement Summary

\begin{tabular}{|c|c|c|c|c|c|c|c|c|c|}
\hline $\begin{array}{c}B \\
(\mathrm{kHz})\end{array}$ & $\begin{array}{c}L_{a} \\
(\mathrm{~dB})\end{array}$ & $\begin{array}{c}\text { Saturn } \\
\operatorname{Var}\end{array}$ & $\begin{array}{c}\mathrm{CMB} \\
\operatorname{Var}\end{array}$ & $\begin{array}{c}C \\
(1 / \mathrm{W})\end{array}$ & $\begin{array}{c}d C \\
(\mathrm{~dB})\end{array}$ & $\begin{array}{c}T_{r} \\
(\mathrm{~K})\end{array}$ & $\begin{array}{c}d T_{r} \\
(\mathrm{~K})\end{array}$ & $\begin{array}{c}T_{r 0} \\
(\mathrm{~K})\end{array}$ & $\begin{array}{c}T_{r f} \\
(\mathrm{~K})\end{array}$ \\
\hline 117 & 17.1 & 73.6 & 67.7 & $1.74 \mathrm{e}+18$ & 0.3 & 1226 & 101 & 558 & 668 \\
117 & 14.2 & 118.6 & 106.7 & $1.83 \mathrm{e}+18$ & 0.3 & 954 & 63 & 558 & 396 \\
117 & 11.3 & 217.2 & 190.3 & $2.10 \mathrm{e}+18$ & 0.2 & 759 & 42 & 558 & 200 \\
117 & 8.4 & 374.9 & 331.2 & $1.74 \mathrm{e}+18$ & 0.2 & 809 & 47 & 558 & 251 \\
468 & 21.1 & 89.8 & 78.5 & $2.11 \mathrm{e}+18$ & 0.2 & 739 & 45 & 558 & 180 \\
468 & 17.1 & 201.1 & 173.7 & $2.02 \mathrm{e} 18$ & 0.2 & 677 & 39 & 558 & 119 \\
935 & 23.1 & 80.1 & 70.5 & $1.42 \mathrm{e}+18$ & 0.2 & 782 & 49 & 558 & 224 \\
935 & 21.1 & 123.7 & 107.9 & $1.48 \mathrm{e}+18$ & 0.2 & 727 & 43 & 558 & 168 \\
935 & 19.1 & 180.0 & 157.5 & $1.32 \mathrm{e}+18$ & 0.2 & 749 & 45 & 558 & 191 \\
935 & 17.1 & 279.7 & 237.0 & $1.57 \mathrm{e}+18$ & 0.2 & 592 & 30 & 558 & 34 \\
4675 & 31.0 & 57.5 & 49.9 & $1.38 \mathrm{e}+18$ & 0.2 & 705 & 40 & 558 & 146 \\
4675 & 27.1 & 130.7 & 111.0 & $1.46 \mathrm{e} 18$ & 0.2 & 600 & 30 & 558 & 41 \\
125000 & 0 & 191712 & 160962 & $1.66 \mathrm{e} 17$ & 0.001 & 558 & 8 & 558 & 0 \\
\hline
\end{tabular}

for a given attenuator and bandwidth selection, then the received counts $V_{\mathrm{n}}$ are given by $V_{\mathrm{n}}=G\left(T_{\mathrm{r}}+T_{\mathrm{a}}\right)$, which has the same form as the radiometer equation. With measurements of two known sources, (7) and (8) can be applied to solve for the radar receiver gain and effective noise temperature, just like the radiometer measurements. When the attenuator is taken as known, then $C$ is determined by such measurements. The uncertainty in $C$ is the uncertainty in $G$, which is driven by the uncertainties in the target temperature and the statistical uncertainty of the receive-only measurements. The uncertainty in $L_{\mathrm{a}}$ is handled separately. Prelaunch measurements of $L_{\mathrm{a}}$ have a standard deviation of $0.2 \mathrm{~dB}$, so the $3 \sigma$ uncertainty is taken to be $0.6 \mathrm{~dB}$. Engineering test data taken during the Saturn tour indicate that $T_{\mathrm{rf}}$ varies with system physical temperature, so the data on $G$ and $T_{\mathrm{r}}$ are most useful if the system is fully warmed up. Table IV shows results obtained using Saturn and the CMB as the two reference targets. These data were obtained on April 18, 2005, as part of an engineering test that had a full 3-h warmup before the data were taken. This kind of passive calibration approach for a radar receiver has also been used on Earth-orbiting radar systems [16].

The radiometer result that supplies $T_{\mathrm{r} 0}$ is also shown in the last line of Table IV. These results illustrate how the receiver temperature increases with decreasing bandwidth, as expected for a narrowband source of contamination in the back end. At higher bandwidths, the receiver temperature tends to the radiometer value as expected. The receiver gain represented by $C$ shows about $1.3 \mathrm{~dB}$ of variability across the different bandwidths. Since each bandwidth goes through a separate filter, this variability is not surprising, but does need to be accounted for when calibrating data from each mode. The onesigma uncertainties shown for $C$ and $T_{\mathrm{r}}$ are derived from the standard deviations of the receive-only variance measurements.

We also see variability in receiver temperature and $C$ as attenuator settings are changed. Table $\mathrm{V}$ shows results obtained during the September 21, 2006 engineering test. Results are shown for all four operating bandwidths using several attenuator settings that are typical values used for each bandwidth. We see that increasing back-end attenuation causes an increase in the receiver noise temperature, which is consistent with the presence of a narrowband back-end noise source. The backend noise source has not been identified, but the power levels in the four active bandwidths at various attenuator settings are measured by engineering tests such as the one summarized by Table V. Variability in this noise source is a key contributor to the overall calibration error budget. When scatterometer data are calibrated, there are noise-only data available, and the value of $C$ can be obtained using the appropriate value of $T_{\mathrm{r}}$ for the attenuator setting used. Most of the scatterometer data are collected using the 8.4 and $14.2 \mathrm{~dB}$ attenuator settings. For SAR data, special noise-only calibration measurements are inserted by RMSS before and after the SAR data collection. These can then be used along with the appropriate $T_{\mathrm{r}}$ value to obtain $C$. In some cases, the noise-only calibration data were lost in data gaps, and an assumed value for $C$ as determined by engineering test data has to be used in the SAR processing.

In the engineering test, the conversion constant $C$ should be constant for a given bandwidth since the gain variations between the on- and off-target measurements are normalized out using the internal resistive load. Both the scatterometer 
TABLE VI

$X^{\prime}$ Main Terms And UnCertainties

\begin{tabular}{|c|c|c|}
\hline Radar Eqn Term & Pre-launch/Typical Value & $3 \sigma$ Uncertaintay $(\mathrm{dB})$ \\
\hline$P_{t}$ & $48.1 \mathrm{~W}$ & 0.73 \\
$G_{0}$ & $50.7 \mathrm{~dB}$ & 0.3 \\
$C$ & $1.3 \mathrm{e} 18$ & 0.8 \\
$L_{a}$ & $8.4 \mathrm{~dB}$ & 0.6 \\
\hline$X^{\prime}$ & varies & 1.3 \\
\hline
\end{tabular}

$(117 \mathrm{kHz})$ and Hi-SAR $(935 \mathrm{kHz})$ data show about $0.8 \mathrm{~dB}$ of variation in $C$ as the attenuators are stepped through some typical values for these modes. The variation is likely due to variation in the effective receiver temperature in between the on- and off-target measurements. The likely culprit is the backend noise since the LNA has shown good noise temperature stability in the radiometer data. Future engineering tests will attempt to address this issue and improve the estimates of $C$. In the meantime, the variability in $C$ has to be assigned to the absolute error budget. The uncertainties in the actual values of $C$ and $T_{\mathrm{r}}$ are higher in Table $\mathrm{V}$ than in Table IV because less integration time and data volume were available, and the measurements are divided across more attenuator settings. Radiometer performance is consistent between these two engineering tests to within $3 \%$.

\section{Statistical Measurement Uncertainty and Noise Subtraction}

In the passive mode and in receive-only measurements conducted in the active bandwidths, the random nature of thermal noise sets the limit on short term measurement stability. The normalized statistical measurement uncertainty is given by [15]

$$
\sigma=\frac{1}{\sqrt{\tau B}}
$$

where $\tau$ is the integration time and $B$ is the noise bandwidth of the measurement. When longer integration times are used, then some attention has to be given to longer term fluctuations. On a longer time scale, extra variance appears to come from small component variations in response to small temperature variations in the system. The resulting gain variation introduces some random-walk noise into the receiver output. The spectral properties of this type of noise have been successfully modeled in other radiometers with a $1 / f$ dependence as shown below [19]

$$
S(f)=a+\frac{b}{f}
$$

where $S$ is the detected power spectrum, $f$ is frequency, and $a$ and $b$ are characteristic constants for a particular receiver system. The quantity $f_{0}=b / a$ is called the knee frequency, where the $1 / f$ noise equals the random thermal noise. At frequencies higher than $f_{0}$, the value of $S(f)$ approaches the usual time-bandwidth result, thus $a=1 / \sqrt{\tau B}$. The knee frequency, $f_{0}$, can be measured for the radar and radiometer receivers by taking data with uniform timing parameters for an extended period of time while observing a fixed target. The

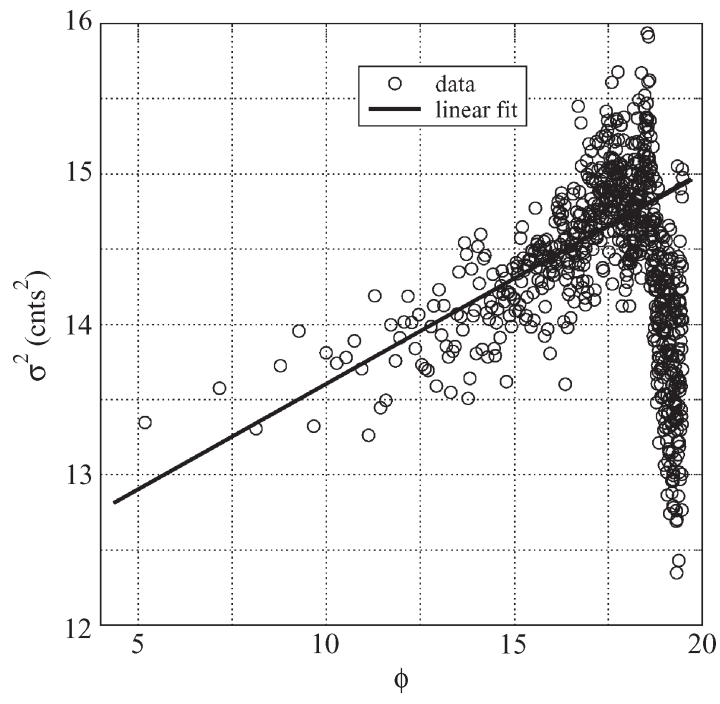

Fig. 12. Two-point variance of radiometer internal resistive load counts as a function of $\phi$, which is related to lag by (22). To remove long-term warmup gain variation, $70 \mathrm{~min}$ of uniform data are first detrended by a linear fit. A linear fit to the measured two-point variance is also shown. The fit only covers lags up to $20 \min (\phi=17.2)$ to avoid contamination by longer term gain variations that need to be removed by the calibration procedure. The plot only shows one in five data points to reduce clutter, but the fit uses all data. The slope of the fit gives a knee frequency $f_{0}=0.01 \mathrm{~Hz}$.

most convenient way to measure $f_{0}$ is to use the two-point variance which is defined by

$$
\sigma^{2}\left(t_{1}, t_{2}\right)=\frac{1}{2}\left\langle\left(s\left(t_{1}\right)-s\left(t_{2}\right)\right)^{2}\right\rangle
$$

where $s\left(t_{1}\right)$ is the signal measurement at time $t_{1}$ and \langle\rangle indicates an expectation operation. We assume that the measurements are uniformly spaced with constant integration time. If we again assume that the data $d(t)$ are samples from a stationary stochastic process, then we can relate the two-point variance of $d(t)$ with its spectral density [19]. If we also assume that the spectral density is given by (19), then we obtain the following theoretical expression for the two-point variance as a function of lag and the knee frequency:

$$
\sigma^{2}(n)=\frac{\langle s\rangle^{2}}{\tau_{\mathrm{p}} B}\left(1+\tau_{\mathrm{b}} f_{0} \phi(n)\right)
$$

where $n$ is the lag expressed as a number of measurement intervals $\tau_{\mathrm{b}}$, which is the burst period for Cassini RADAR, $\tau_{\mathrm{p}}$ is the integration time, $B$ is the measurement bandwidth, and $\phi(n)$ is the following function of lag:

$$
\begin{aligned}
\phi(n)=(n-1)^{2} \log (n-1)- & 2 n^{2} \log n \\
& +(n+1)^{2} \log (n+1) .
\end{aligned}
$$

Note that the two-point variance $\sigma^{2}(n)$ is a logarithmic function of lag and a linear function of $\phi(n)$.

The two-point variance of the data $s(t)$ as a function of lag can be computed directly from the definition in (20). Since we are assuming stationary stochastic data, the expectation averages over all $t_{1}$ and $t_{2}$ that are separated by the same lag time $t=t_{1}-t_{2}$. Figs. 12 and 13 show the two-point variances computed from $70 \mathrm{~min}$ of radiometer data and $70 \mathrm{~min}$ of radar data, respectively as a function of $\phi$. These data were obtained 


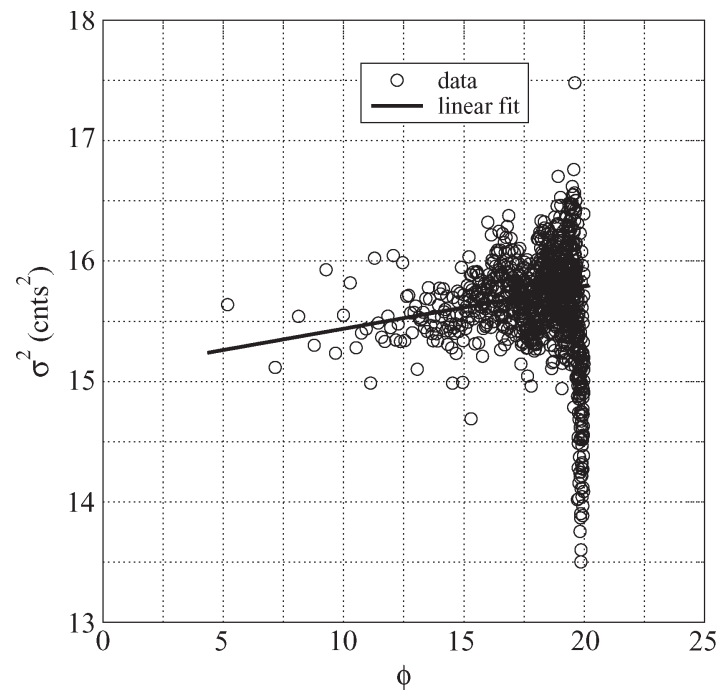

Fig. 13. Two-point variance of radar receiver in scatterometer mode as a function of $\phi$ which is related to lag by (22). To remove long-term warmup gain variation, $70 \mathrm{~min}$ of uniform data are first detrended by a linear fit. A linear fit to the measured two-point variance is also shown. The fit only covers lags up to $20 \min (\phi=17.7)$ to avoid contamination by longer term gain variations that need to be removed by the calibration procedure. The plot only shows one in five data points to reduce clutter, but the fit uses all data. The slope of the fit gives a knee frequency $f_{0}=0.003 \mathrm{~Hz}$.

during a long observation of Iapetus on January 1, 2005. A linear warmup trend was removed before computing the twopoint variance to avoid contamination of the long lag values. Also shown in these plots are linear least squares fits. The fits use only lags up to $20 \mathrm{~min}$ to avoid contaminating the result with longer term gain variations that have to be calibrated out using reference measurements. The slope of the fit is related to $f_{0}$, as shown in (21). The knee frequency obtained for the radiometer is about $0.01 \mathrm{~Hz}$, which implies measurement stability for about $100 \mathrm{~s}$ before $1 / f$ noise begins to intrude. In practice, all radiometer integrations are $4 \mathrm{~s}$ or less, and $1 / f$ noise is not a significant issue. The knee frequency obtained for the radar receiver operating in scatterometer mode is about $0.003 \mathrm{~Hz}$, which corresponds to measurement stability for about 5.5 min. Regular Titan observations rely on integration times well below this value. Most of the icy satellite integrations also run for less time than this value. A few run longer, but the measurement variance is not a significant factor in these cases. Icy satellite albedo uncertainties are instead dominated by calibration uncertainties and systematic effects in fitting a surface model function.

The stability of the radar and radiometer receivers are actually much better than the stability of the engineering model, which was also tested on the ground, resulting in a knee frequency of about $0.1 \mathrm{~Hz}$ and a stability time of about $10 \mathrm{~s}$. This probably reflects the thermal stability of the spacecraft environment. Cassini is a very large spacecraft, and Saturn is located far from the sun. Once the radar receiver warms up, there are few varying heat sources that could perturb it thermally.

In the active modes, the received signal power $P_{\mathrm{s}}$ is the quantity of interest. The instrument actually measures $P_{\mathrm{r}}=$ $P_{\mathrm{s}}+P_{\mathrm{n}}$, which is the sum of the echo signal power and thermal noise from the system and from target emission. $P_{\mathrm{s}}$ is related to the scattering characteristics of the target as discussed in the next section on the radar equation. The signal-only data counts $V_{\mathrm{s}}$ are estimated by subtracting an estimate of the noise from a measurement of the signal plus noise

$$
V_{\mathrm{s}}=V_{\mathrm{sn}}-V_{\mathrm{n}}
$$

where $V_{\mathrm{sn}}$ is the variance of the data counts in the echo buffer, and $V_{\mathrm{n}}$ is the variance of the noise-only counts.

During scatterometry mode data collections, it is possible to measure both $V_{\mathrm{sn}}$ and $V_{\mathrm{n}}$ by segmenting out the noiseonly portions of the data in the time domain or the frequency domain. In these cases, the uncertainty in $V_{\mathrm{s}}$ comes from the statistical uncertainty of the two measurements $V_{\mathrm{sn}}$ and $V_{\mathrm{n}}$ as given by (3). For Titan scatterometry scans, the timing parameters give a normalized standard deviation of about $0.2 \mathrm{~dB}$ for $V_{\mathrm{s}}$. For distant icy satellite scatterometry observations, the disk integrated measurements are limited by $1 / f$ noise to a normalized standard deviation of about $1 \%(0.04 \mathrm{~dB})$.

During SAR and altimetry data collections, there is generally no noise-only data to be segmented out. In these cases, the noise level is estimated using (16). The values of $C$ and $T_{\mathrm{r}}$ used should correspond to the attenuator and bandwidth setting used for a particular measurement. The coincident radiometer measurement can be conveniently used to supply $T_{\mathrm{a}}$. The uncertainty in $V_{\mathrm{sn}}$ will be lower for SAR and altimetry compared to scatterometry because of the higher time bandwidth product. This will lead to less uncertainty for $V_{\mathrm{S}}$ in most SAR and altimetry situations where SNR is relatively high and the uncertainty in $V_{\mathrm{n}}$ is relatively less important. Producing real aperture results from SAR data also requires a correction for BAQ bias which is caused by noise only intervals in the data used to compute BAQ encoding coefficients. Using simulated data the following correction was derived and applied to real aperture calculations for imaging data using 8- to 2-bit BAQ encoding:

$$
V_{\mathrm{sn}}^{\prime}=\frac{V_{\mathrm{sn}}}{f_{\mathrm{baq}}}
$$

where $V_{\mathrm{sn}}^{\prime}$ is the corrected echo buffer variance to use in (23) and

$$
f_{\text {baq }}=-2.3936+5.7853 \frac{M_{8}}{M}-2.430\left(\frac{M_{8}}{M}\right)^{2}
$$

where $M_{8}$ is the average magnitude of the data in the first and last eight PRI intervals of the echo buffer which are used by the BAQ encoding algorithm [9], and $M$ is the average magnitude of the echo buffer excluding the first and last eight PRI intervals. The ratio $M_{8} / M$ is restricted to the range $[0.77,1.026]$ before using (25). As discussed earlier, altimetry data is collected with a surplus of transmitted pulses and does not suffer from BAQ bias.

\section{E. Radar Equation and Error Budget}

Ultimately, we are interested in the properties of the illuminated target area, not the power received by the radar. When measuring a surface with a radar, the most commonly used quantity is the normalized backscattering cross section $\sigma_{0} . \sigma_{0}$ is determined by the detailed dielectric and geometric 
characteristics of the many individual scatterers in the illuminated area. It is related to the received power by the radar equation [17, Sec. 7.16]

$$
P_{\mathrm{S}}(t)=\frac{\lambda^{2}}{(4 \pi)^{3}} \int_{A} \frac{P_{\mathrm{t}}\left(t-\frac{2 R}{c}\right) u_{\mathrm{rw}}(t) G^{2} \sigma_{0}}{R^{4}} d A
$$

where $P_{\mathrm{s}}(t)$ is the received signal power referenced at the antenna port at time $t, \lambda$ is the transmitted wavelength, $A$ is the area on the surface illuminated by the antenna main lobe, $P_{\mathrm{t}}(t)$ is the radiated transmit power pulse train, $u_{\mathrm{rw}}(t)$ is the receive window filter, $G$ is the antenna gain (neglecting transmit receive differences due to spacecraft motion during the round trip time), $\sigma_{0}$ is the normalized backscattering cross section, and $R$ is the range to the surface. The receive window filter is defined by

$$
u_{\mathrm{rw}}(t)= \begin{cases}1, & \text { for } \tau_{\mathrm{rwd}}<t<\tau_{\mathrm{rwd}}+\tau_{\mathrm{rw}} \\ 0, & \text { otherwise }\end{cases}
$$

where $\tau_{\text {rwd }}$ is the delay from the start of a transmitted pulse train to the time the receive window is opened and the receiver begins digitizing echo power, and $\tau_{\mathrm{rw}}$ is the duration of the echo buffer (both set by the current IEB instruction). The receive window filter selects out the time during which received power is actually recorded and forces an accounting of any power lost due to a mispositioned receive window or due to range spreading of echos at higher incidence angles. The general radar equation specializes in different ways for the different observing circumstances encountered by the Cassini RADAR.

During Titan flybys the target disk is much larger than the beam main lobe, so we can approximate the surface as locally flat within the beam at a fixed incidence angle $\theta_{\mathrm{i}}$ and range $R_{0}$ specified at the boresight. For altitudes typical of a scatterometry scan $(25000 \mathrm{~km})$, this approximation begins to fail at incidence angles above $65^{\circ}$ where the error in backscatter increases above $0.1 \mathrm{~dB}$. At lower incidence angles the range and backscatter cross section can be taken as constant and pulled out of the integral. The pulse train is given by

$$
\begin{gathered}
p(t)= \begin{cases}1, & \text { for } 0<t<\tau_{\mathrm{p}} \\
0, & \text { otherwise }\end{cases} \\
P_{\mathrm{t}}(t)=P_{\mathrm{t} 0} \sum_{i=0}^{N_{\mathrm{p}}-1} p\left(t-i \tau_{\mathrm{pri}}\right)
\end{gathered}
$$

where $P_{\mathrm{t} 0}$ is the transmit power level during a pulse transmission, $N_{\mathrm{p}}$ is the number of pulses transmitted in a burst, and $\tau_{\mathrm{pri}}$ is the pulse repetition interval. For real aperture results, we need to average the received signal power over the time of the echo buffer in the same way that the echo buffer data are averaged when forming $V_{\mathrm{s}}$. The average received signal power $P_{\mathrm{s}}$ is given by

$$
P_{\mathrm{s}}=\frac{1}{\tau_{\mathrm{rw}}} \int_{0}^{\tau_{\mathrm{rw}}} P_{\mathrm{s}}(t) d t
$$

Substituting (26) and (29) into (30), applying the locally flat approximation, and reversing the order of integration gives

$$
\begin{aligned}
P_{\mathrm{s}}= & \frac{\lambda^{2}}{(4 \pi)^{3}} \frac{P_{\mathrm{t} 0} \sigma_{0}\left(\theta_{\mathrm{i}}\right)}{R_{0}^{4}} \frac{1}{\tau_{\mathrm{rw}}} \\
& \times \int_{A} \int_{0}^{\tau_{\mathrm{rw}}} \sum_{i=0}^{N_{\mathrm{p}}-1} p\left(t-i \tau_{\mathrm{pri}}-\frac{2 R}{c}\right) u_{\mathrm{rw}}(t) G^{2} d t d A .
\end{aligned}
$$

If the receive window covers all of the echo power including range spread, then the time integral over the pulse train summation can be simplified to the number of pulses $N_{\mathrm{p}}$ multiplied by the integral over one pulse. Here, we also assume that the echo powers from each pulse add up independently. This assumption is generally true even though the echo power from the end of one pulse partially overlaps the echo power from the beginning of the next pulse in many of the radar observations. In (31), the relatively long chirped pulses are convolved with the antenna gain pattern. For portions of the echo buffer, there are often two adjacent pulses contributing echo power at the same time. The fields are uncorrelated because these contributions sample different portions of the chirp waveform, and their powers still add up in the echo buffer. The time integral over one pulse then evaluates to just $\tau_{\mathrm{p}}$, and we have

$$
P_{\mathrm{s}}=\frac{\lambda^{2}}{(4 \pi)^{3}} \frac{P_{\mathrm{t} 0} \sigma_{0}\left(\theta_{\mathrm{i}}\right)}{R_{0}^{4}} \frac{N_{\mathrm{p}} \tau_{\mathrm{p}}}{\tau_{\mathrm{rw}}} \int_{A} G^{2} d A .
$$

When the echos from the transmitted pulse train are not fully captured by the receive window, then a correction factor $f_{\text {rcv }}$ for the average fraction of pulses actually received needs to be computed from the viewing geometry, the beamwidth, and the time position of the receive window. Applying this factor and evaluating the area integral using a Gaussian main lobe with the width shown in Table II gives

$$
P_{\mathrm{s}}=\frac{\lambda^{2}}{(4 \pi)^{3}} \frac{P_{\mathrm{t}} G_{0}^{2}}{R_{0}^{4}} \frac{N_{\mathrm{p}} \tau_{\mathrm{p}} f_{\mathrm{rcv}}}{\tau_{\mathrm{rw}}} \sigma_{0}\left(\theta_{\mathrm{i}}\right) \frac{A}{2 \ln 2}
$$

where $G_{0}$ is the antenna peak gain referenced at the antenna port, $A$ is the area of the beam projected on the surface

$$
A=\frac{\pi\left(R_{0} \frac{\theta_{\mathrm{bw}}}{2}\right)^{2}}{\cos \theta_{\mathrm{i}}}
$$

$\sigma_{0}$ is assumed to be azimuthally symmetric, and $R_{0}$ is the range to the boresight intercept point. The factor of $1 /(2 \ln 2)$ comes from integrating the squared Gaussian main lobe of the gain pattern. Equation (33) applies to most of the active mode data collected on Titan flybys. In a few cases, there are data collected from high incidence angles near the limb of the target body. Here, the locally flat approximation breaks down, and the radar equation needs to be numerically integrated to obtain the correct scaling for $\sigma_{0}$.

The terms multiplying $\sigma_{0}$ are now lumped together into one factor called $X$ so that

$$
\sigma_{0}=\frac{P_{\mathrm{s}}}{X}
$$


and the uncertainty in $\sigma_{0}$ is the sum of the uncertainties in $X$ and $P_{\mathrm{s}}$. If we further lump the receiver gain into a modified factor called $X^{\prime}$ then

$$
\begin{aligned}
X^{\prime} & =\frac{C X}{L_{\mathrm{a}}} \\
\sigma_{0} & =\frac{V_{\mathrm{s}}}{X^{\prime}} .
\end{aligned}
$$

Table VI summarizes the main factors that go into $X^{\prime}$ along with their corresponding prelaunch values and uncertainties. If we assume that these error sources are independent, the total calibration error is calculated by root-sum-squaring the uncertainties from each of the multiplicative terms that make up $X^{\prime}$.

During distant Titan and icy satellite observations, the target apparent angular spread may be smaller than the beam main lobe, so the illuminated area is the visible disk of the target body. Under these conditions, the radar equation can be restated in a more specialized form

$$
P_{\mathrm{s}}=\frac{\lambda^{2}}{(4 \pi)^{3}} \frac{P_{\mathrm{t}} G_{0}^{2} R_{\mathrm{s}}^{2}}{0 R_{0}^{4}} \frac{N_{\mathrm{p}} \tau_{\mathrm{p}}}{\tau_{\mathrm{rw}}} \int_{0}^{\theta_{\max }} \int_{0}^{2 \pi} g(\theta, \phi)^{2} \sigma_{0}(\theta) \sin \theta d \theta d \phi
$$

where $R_{\mathrm{S}}$ is the target radius (approximating as a sphere), $R_{0}$ is the range to the center of the target body, $\theta_{\max }=a \tan \left(R / R_{\mathrm{s}}\right)$ is the angle to the limb, $g$ is the normalized antenna pattern (from sun-scan data), and $\theta, \phi$ are standard spherical coordinates centered on the target body. This specialized radar equation assumes that $R \gg R_{\mathrm{s}}$, which leads to $\theta \approx \theta_{\mathrm{i}}$ and $R \approx R_{0}$. In these cases, the number of pulses transmitted is large enough that the receive window captures most of the echo power and $f_{\mathrm{rw}} \approx 1$.

\section{F. Relative and Absolute Calibration Performance}

Part of the absolute calibration error of $1.3 \mathrm{~dB}$ for the Cassini RADAR comes from uncertainties in the transmit power and the attenuator setting. Unlike the radiometer mode, which can be fully calibrated against well-known sources, the active modes ultimately require these prelaunch measurements since there are no known reference targets in the Saturn system. During the Earth flyby in August 1999, some scatterometry data over the Pacific Ocean and South America were obtained. However, comparing Cassini results with other Earth-orbiting sensors required model-based extrapolation to line up the position, incidence angle, and frequency of the results [18]. The results were good for validation and relative observation, but not for precision absolute calibration. Eventually, distant Titan scatterometry observations made by the Cassini RADAR may be compared with Earth-based radar measurements of Titan to tighten the absolute calibration. The receiver gain uncertainty may be improved in the future by better engineering tests and improved calibration of the radiometer.

The absolute calibration uncertainty is not the only error of potential interest. Relative calibration uncertainties describe how changes in system performance can change measurements taken at different times. Fortunately, the Cassini RADAR has shown good stability once the system has warmed up fully, which takes about $3 \mathrm{~h}$. The system is stable enough that relative calibration uncertainties can be much smaller than the absolute calibration uncertainty. Within one scatterometry scan of Titan during a Titan flyby, if a single attenuator setting is used, then the error terms in $X^{\prime}$ become very small because these quantities do not drift on short time scales (minutes). In this case, the relative uncertainty between $\sigma_{0}$ measurements is dominated by statistical measurement uncertainty $\left(K_{\mathrm{pc}}\right)$, which is about $0.6 \mathrm{~dB}(3 \sigma)$ for scatterometry scans. Attenuator changes will increase the uncertainty; however, the attenuator step uncertainty can often be reduced by examining noise-only data across the step and applying the measured gain change. In the SAR and altimetry modes, this is not possible due to the use of BAQ encoding, so attenuator steps will bump up relative errors within a swath to $0.8 \mathrm{~dB}$. Relative errors between different Titan flybys are set by the uncertainty in $C$, while assuming that $P_{\mathrm{t}}$ and the attenuator loss factors remain unchanged. Since the one-sigma measurement uncertainty in $C$ is about $0.3 \mathrm{~dB}$, the three-sigma relative uncertainty between Titan flybys is about $0.9 \mathrm{~dB}$.

Relative uncertainties between icy satellite scatterometry measurements are determined largely by the uncertainty in receiver gain $C$ at the 8.4-dB attenuator setting. This attenuator setting is low enough that the receiver temperature is dominated by the front-end. Radiometer measurements of Saturn have shown the front-end receiver temperature to be stable to within about $10 \%$, which implies similar relative precision for the icy satellite backscatter measurements. In some cases, pointing uncertainties may become significant because the beam main lobe is very close to the limb where small pointing errors can make a large difference in the illuminated area.

Finally, we should remember that the total uncertainty in $\sigma_{0}$ comes from the combination of the absolute calibration uncertainty in $X^{\prime}$ and the statistical uncertainty $\left(K_{\mathrm{pc}}\right)$ in the measurement $V_{\mathrm{s}}$. Real aperture measurements usually have $K_{\mathrm{pc}}$ levels lower than the calibration uncertainty in $X^{\prime}$, and the latter dominates. In SAR images, the statistical noise in each pixel can be larger than the calibration uncertainty in $X^{\prime}$, and the total uncertainty of an individual pixel can be many decibels. This occurs, in particular, when the number of looks is low (at Titan periapsis and during ride-along observations) or when the SNR is particularly low (in the outer beams at the ends of a swath or in radar dark regions, such as methane lakes). The statistical uncertainty can be reduced at the expense of resolution by averaging adjacent pixels together while the calibration uncertainty remains constant.

\section{Data Processing}

Once data are obtained from the Cassini RADAR, they are run through a preprocessor, which produces data files consisting of burst-ordered data records. Two types of burst-ordered data products are generated. Short burst data records (SBDRs) are produced for all radar bursts. Long burst data records (LBDRs) are produced for all radar bursts that use an active mode. These append an echo data buffer to each record that contains the raw BAQ-decoded echo data from the science data buffer. SBDRs are relatively small because they do not contain the 
echo data. Both SBDR and LBDR files have a standard text header that describes the data file and specifies the time span included. Each record in an SBDR file contains three levels of data. The first segment includes all of the IEB parameters and all of the instrument telemetry measured in flight. The raw radiometer integration measurements are also included here. The preprocessor converts these measurements to standard metric units, where appropriate. The second segment contains quantities that describe the timing and viewing geometry. Quantities such as the spacecraft position and velocity are reported in inertial (J2000) coordinates and in target body-fixed coordinates. These are derived from ephemeris files supplied by the spacecraft navigation team. Some quick-look products are generated right after a flyby using predicted ephemeris files, and then replaced by final products several weeks later when reconstructed ephemeris files are available. The third segment reports science results depending on the mode of operation. Radiometer data are processed into an uncalibrated antenna temperature. When the SBDR products are delivered to the planetary data system (PDS), an associated text file describes the final calibration factor that needs to be applied to the uncalibrated antenna temperatures to produce a calibrated antenna temperature. This procedure allows the radiometer calibration to be refined during the mission. Scatterometer processing is performed at Stanford University. Details are available in [20]. The main output of scatterometer processing is the real aperture normalized backscattering cross section $\left(\sigma_{0}\right)$ as given by (35). This quantity can be computed for all active modes. Altimeter data are processed under the sponsorship of the ASI. The resulting height above the reference spheroid is placed in a field in the SBDR and LBDR files. A special product called an altimeter burst data record is also generated, which contains a range-compressed waveform for each burst. The waveform is produced as a standard product because it provides a rich data set for future analysis of the nature of Titan's surface. The highrange resolution supports modeling of the surface structure as in [21].

SAR processing is performed at the Jet Propulsion Laboratory (JPL) using a modified SPECAN algorithm. SPECAN stands for Spectral Analysis. This algorithm is often used on radar systems where the synthetic aperture is relatively short. The core of the algorithm consists of three steps: range compression, deramping operation, and finally FFT which separates the data by Doppler frequency in azimuth. More details about the SPECAN algorithm are available in [22, Ch. 9]. Processing of SAR data from a Titan flyby is also complicated by the rapidly varying look geometry during the data take. Details of the special challenges of processing flyby SAR data are described in [23]. SAR processing produces raster image products in a special PDS file type called a basic image data record (BIDR) modeled on Magellan data products. A BIDR file is a 2-D array of 32-bit floating point values stored in binary form. A standard text header is prepended at the start of the file. The header includes data defining the oblique cylindrical coordinate system used to represent the swath image data. The main product is the $\sigma_{0}$ BIDR file, which contains the swath image. Each pixel in this file is a calibrated $\sigma_{0}$ value. A noise subtracted BIDR file is also provided, which removes an estimate of the thermal and quantization noise power from each pixel. The SAR processor also outputs additional BIDR format files that contain intermediate results of interest at the pixel level. These include the incidence angle and the starting and ending burst numbers that provided data for the corresponding image pixel. Additional higher order map products are produced by the U.S. Geological Survey and are also delivered to the PDS.

The files delivered to the PDS are organized in volumes, where each volume contains data obtained from the execution of one IEB. Usually, this represents one flyby, or one target. In addition to the SBDR, LBDR, and BIDR files, the volume also includes beam pattern files and various documentation files that describe the specific observation and the detailed format of the data files. Data volumes are delivered to the PDS one year after acquisition and can be accessed at pds.nasa.gov.

\section{REFERENCES}

[1] C. Elachi et al., "RADAR: The Cassini Titan radar mapper," Space Sci. Rev., vol. 115, no. 1-4, pp. 71-110, Nov. 2004.

[2] D. Campbell et al., "Radar evidence for liquid surfaces on Titan," Science, vol. 302, no. 5644, pp. 431-434, Oct. 2003.

[3] D. Muhleman et al., "Radar investigation of Mars, Mercury, and Titan," Annu. Rev. Earth Planet. Sci., vol. 23, pp. 337-374, May 1995.

[4] S. Ostro, "Planetary radar astronomy," Rev. Mod. Phys., vol. 65, no. 4, pp. 1235-1279, 1993.

[5] D. Muhleman et al., "Radar reflectivity of Titan," Science, vol. 248, no. 4958 , pp. $975-980$, May 1990.

[6] M. Fulchignoni et al., "In situ measurements of the physical characteristics of Titan's environment," Nature, vol. 438, no. 7069, pp. 785-791, Dec. 2005.

[7] J. Curlander and R. McDonough, Synthetic Aperture Radar. Hoboken, NJ: Wiley, 1991.

[8] R. Kwok and W. T. K. Johnson, "Block adaptive quantization of Magellan SAR data," IEEE Trans. Geosci. Remote Sens., vol. 27, no. 4, pp. 375383, Jul. 1989.

[9] P. Renick, Cassini RADAR Digital Assembly (DSS) High Level Design, 1997. JPL Doc. JPL-D-11201.

[10] H. Zebker et al., "Titan's surface from reconciled Cassini microwave reflectivity and emissivity observations," Icarus, vol. 194, no. 2, pp. 704 710, Apr. 2008

[11] M. Spencer, C. Wu, and D. G. Long, "Improved resolution backscatter measurements with the seawinds pencil-beam scatterometer," IEEE Trans. Geosci. Remote Sens., vol. 38, no. 1, pp. 89-104, Jan. 2000.

[12] M. Janssen et al., "Titan's surface at $2.2-\mathrm{cm}$ wavelength imaged by the Cassini RADAR radiometer: Calibration and first results," Icarus, 2009. in press.

[13] W. Imbriale, Spaceborne Antennas for Planetary Exploration. New York: Wiley-Interscience, 2006.

[14] S. Brown, S. Desai, W. Lu, and A. B. Tanner, "On the long-term stability of microwave radiometers using noise diodes for calibration," IEEE Trans. Geosci. Remote Sens., vol. 45, no. 7, pp. 1908-1920, Jul. 2007.

[15] F. Ulaby et al., Microwave Remote Sensing, vol. I. Reading, MA: Addison-Wesley, 1981.

[16] N. Pierdicca, B. Greco, C. Bignami, P. Ferrazzoli, V. Mattioli, and L. Pulvirenti, "The calibration of the ENVISAT radar altimeter receiver by a passive technique," IEEE Trans. Geosci. Remote Sens., vol. 44, no. 11, pp. 3297-3307, Nov. 2006.

[17] F. Ulaby et al., Microwave Remote Sensing, vol. II. Reading, MA: Addison-Wesley, 1982

[18] R. Lorenz, "Cassini Radio Detection and Ranging (RADAR): Earth and Venus observations: First results from Cassini: Venus and Earth swing-bys," J. Geophys. Res., vol. 106, no. A12, pp. 30271-30279, 2001.

[19] M. Janssen, 1996. Unpublished paper

[20] L. Wye et al., "Electrical properties of Titan's surface from Cassini RADAR scatterometer measurements," Icarus, vol. 188, no. 2, pp. $367-$ 385, Jun. 2007.

[21] G. Franceschetti, P. S. Callahan, A. Iodice, D. Riccio, and S. D. Wall, "Titan, fractals, and filtering of Cassini altimeter data," IEEE Trans. Geosci. Remote Sens., vol. 44, no. 8, pp. 2055-2062, Aug. 2006.

[22] I. Cumming and F. Wong, Digital Processing of Synthetic Aperture Radar Data. Norwood, MA: Artech House, 2005.

[23] B. Stiles et al., "Ground processing of Cassini RADAR imagery of Titan," in Proc. IEEE Conf. RADAR, Verona, NY, Apr. 22-27, 2006. 


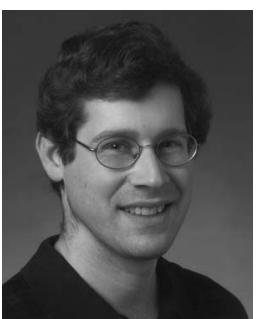

Richard D. West received the Ph.D. degree in electrical engineering from the University of Washington, Seattle, in 1994. His Ph.D. thesis applied dense medium scattering theory to the analysis of passive microwave measurements of Antarctic snow.

Since 1995, he has been with the Radar Science and Engineering Section, Jet Propulsion Laboratory, California Institute of Technology, Pasadena. He has worked on the development of algorithms for the processing and calibration of data from the NASA Scatterometer, and from SeaWinds on QuikScat. In 1999, he began working with the radar instrument on the Cassini Mission to Saturn, and became the Deputy Task Manager in 2002. From 2002 to 2004, he coordinated a small team that developed software for radar sequence generation and for data processing. During the Cassini Prime Mission from 2004 to 2008, he worked on all aspects of instrument operations including planning the science observations in coordination with the science team, designing the radar command sequences, and processing/calibrating the data received. His research interests include electromagnetic scattering theory, the applications of active and passive microwave data to problems in remote sensing and planetary science, and the development of new techniques/technology to enable more capable remote sensing missions.

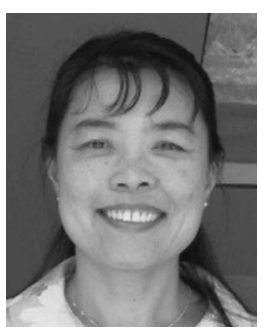

Yanhua Anderson received the B.S. degree in seismology from Beijing University, Beijing, China, in 1983 and the Ph.D. degree in geophysics from the University of Chicago, Chicago, IL, in 1997.

Since 2000, she has been a Science Systems Operation Engineer with the Jet Propulsion Laboratory, California Institute of Technology, Pasadena. She was a Science Planner and Operator for the Galileo Millennium Mission. She currently works for a team of scientists and engineers to plan and sequence the radar commands sent to the Cassini spacecraft.

Rudy Boehmer, photograph and biography not available at the time of publication.

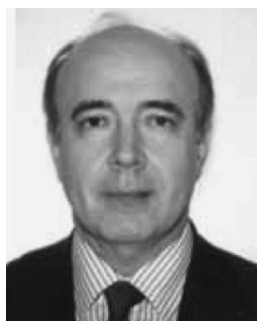

Leonardo Borgarelli received the degree in electronical engineering from the University of Rome "La Sapienza," Rome, Italy.

Since 1985, he has been with Thales Alenia Space Italia, Rome, in the remote sensing field. He is currently Responsible of the Synthetic Aperture Radar (SAR) Group in Rome. He cooperates with universities on feasibility studies for new spaceborne techniques. He has contributed to the awarding of the international competition on KOMPSAT-5 SAR, where was the Senior Technical Advisor. He has been Project Leader in the SAR2000 Programme for the technological development of the Imaging SAR using an active phased array antenna developing the qualification model of the radar embarked on the COSMO Skymed mission for the Italian Space Agency (ASI). He has been leader of the Outdoor Verification Test whose objective was the experimental verification of the radar performance on calibrated targets through the inverse SAR technique. In cooperation between NASA-ASI, within the European Space Agency (ESA) Mars Express mission, he has been Deputy Experiment Manager for the definition and development of the first operational spaceborne Radar Sounder (MARSIS) in the MARS Express Mission. He has been Project Manager of the Radar Altimeter 2 (RA-2) within the Environmental Satellite Program of the ESA. He has been the System Engineer of the Multimode Titan Radar Mapper in cooperation between NASA-ASI for the Cassini deep space mission. He has been System Analysis Engineer for the performance prediction and evaluation of the ESA European Remote Sensing Satellite-1 RA.

Philip Callahan, photograph and biography not available at the time of publication.

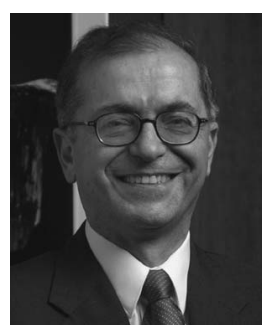

Charles Elachi received the B.Sc. degree in physics from the University of Grenoble, Grenoble, France, in 1968, the Dipl.Ing. degree in engineering from the Polytechnic Institute, Grenoble, in 1968, the M.Sc. and $\mathrm{Ph} . \mathrm{D}$. degrees in electrical sciences from the California Institute of Technology, Pasadena, in 1969 and 1971, respectively, the MBA degree from the University of Southern California, Los Angeles, in 1979, and the M.Sc. degree in geology from the University of California, Los Angeles, 1983.

Since 1970, he has been with Jet Propulsion Laboratory (JPL), California Institute of Technology, Pasadena, first as the Director for Space and Earth Science Programs in 1982, where he was responsible for the development of numerous flight missions and instruments for Earth observation, planetary exploration, and astrophysics, and since May 2001, as the Director of the Jet Propulsion Laboratory, where he is responsible for the planetary and space exploration work of 5000 employees and a budget of $\$ 1.4$ billion.

Dr. Elachi was elected to the National Academy of Engineering in 1989 and has served on a number of academy committees. He has received numerous awards and is author of numerous papers in the fields of active microwave remote sensing and electromagnetic theory.

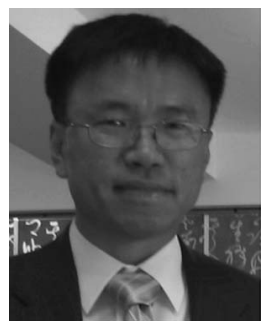

Yonggyu Gim received the Ph.D. degree in physics from the University of Maryland, College Park, in 1996.

He had worked on the design and testing of a magnetic microscope using a high-temperature superconducting quantum interference device. He was with the Los Alamos National Laboratory, Los Alamos, NM. Since 2000, he has been with the Jet Propulsion Laboratory, California Institute of Technology, Pasadena. He initially started working on the radar performance analysis of Cassini RADAR for synthetic aperture radar (SAR) imaging of Titan's surface. During the Cassini prime mission period, he designed the spacecraft attitude for SAR imaging and also got involved in various aspects of Cassini RADAR mission such as radar command design, uplink/downlink processors, and altimeter/radiometer processor developments. His current research interests include radar sounding of Mars and Earth glacier fields, electromagnetic propagation inside a small object like an asteroid, and digital signal processing.

Gary Hamilton, photograph and biography not available at the time of publication.

Scott Hensley received the B.S. degrees in mathematics and physics from the University of California, Irvine, and the Ph.D. degree in mathematics from the State University of New York, Stony Brook, where he specialized in the study of differential geometry.

Subsequent to graduating, he was with Hughes Aircraft Company on a variety of radar systems, including the Magellan radar. Since 1992, he has been with the Jet Propulsion Laboratory, Pasadena, CA, where he studies advanced radar techniques for geophysical applications. His research has involved using both stereo and interferometric data acquired by the Magellan spacecraft at Venus. He was with the European Remote Sensing Satellite-1, Japanese Earth Resources Satellite-1, and Spaceborne Imaging Radar-C data for differential interferometry studies of earthquakes and volcanoes. His current research also includes studying the amount of penetration into the vegetation canopy using simultaneous L- and C-band topographic synthetic aperture radar measurements and repeat-pass airborne interferometry data collected at lower frequencies (P-band). He was the Geographic Synthetic Aperture Radar Chief Scientist, a simultaneous X-band and P-band radar interferometer for mapping above and beneath the canopy that is currently commercially operated by Earthdata International. He was the technical lead of the Shuttle Radar Topography Mission Interferometric Processor Development Team that was a Shuttle-based interferometric radar used to map the Earth's topography between $\pm 60^{\circ}$ latitude. He is currently the Principal Investigator the Uninhabited Aerial Vehicle Synthetic Aperture Radar NASA program that is developing a repeatpass radar interferometry capability for use on conventional or unmanned aerial vehicles. Recently, he began working with the Goldstone Solar System Radar to generate topographic maps of the lunar surface. 


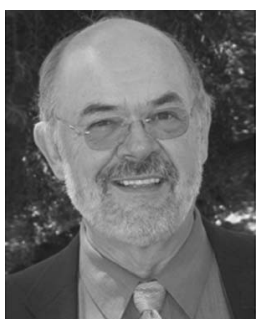

Michael A. Janssen received the B.S. degree in atmospheric and space sciences and the Ph.D. degree in physics from the University of California, Berkeley in 1963 and 1972, respectively.

$\mathrm{He}$ is currently with the Jet Propulsion Laboratory, California Institute of Technology, Pasadena. $\mathrm{He}$ has over 30 years of experience in radio astronomy and has specialized in the microwave remote sensing of the Earth and solar system, observational astrophysics and cosmology, and the development and application of microwave instrumentation for astrophysics and remote sensing. He was a member of the Science Working Group for the Cosmic Background Explorer (COBE) and played a major role in implementing the COBE Differential Microwave Radiometer experiment on COBE that discovered the origin of structure in the universe. In the same period, he edited a book on the microwave remote sensing of atmospheres that is widely used in the field. In addition, serving as lead for the Cassini RADAR radiometer effort, he is currently a Co-Investigator on the Rosetta Microwave Instrument for the Rosetta Orbiter investigation and Principal Investigator for the Juno Microwave Radiometer, scheduled for launch in 2011.

William T. K. Johnson, photograph and biography not available at the time of publication.

Kathleen Kelleher, photograph and biography not available at the time of publication.

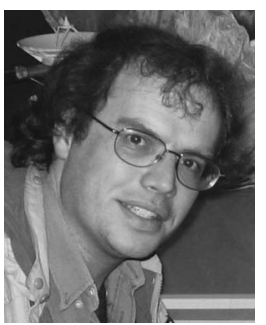

Ralph Lorenz received the B.Eng. degree in aerospace systems engineering from the University of Southampton, Southampton, U.K., and the Ph.D. degree in physics from the University of Kent, Canterbury, U.K., in 1994.

From 1990 to 1991, he was with the European Space Agency on the design of the Huygens probe and during his Ph.D. research designed and built its penetrometer instrument that 12 years later measured the mechanical properties of Titan's surface when Huygens landed in January 2005. From 1994 to 2006, he worked as a Planetary Scientist in the Lunar and Planetary Laboratory, University of Arizona, Tucson, with particular interests in Titan, Mars, planetary climate, nonequilibrium thermodynamics, aerospace vehicles, and radar. He continues to work on those topics in the Applied Physics Laboratory, Johns Hopkins University, Laurel, MD. He is on the editorial board of the International Journal of Astrobiology and is author or coauthor of several books including Lifting Titan's Veil, Spinning Flight, and Space Systems Failures as well as over 140 publications in refereed journals.

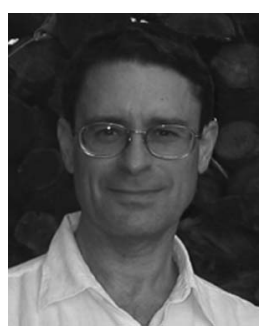

Steve Ostro (M'07) received the B.S. degree in ceramic science from Rutgers University, Camden, NJ, in 1969, the M.S. degree in engineering physics from Cornell University, Ithaca, NY, in 1974, and the $\mathrm{Ph} . \mathrm{D}$. degree in planetary science from the Massachusetts Institute of Technology, Cambridge, in 1978.

From 1979 to 1984, he worked as an Assistant Professor of astronomy with Cornell University and has been with the Jet Propulsion Laboratory, California Institute of Technology, Pasadena, since 1984, where he is currently a Senior Research Scientist, heads the Asteroid Radar group, and works on the Cassini-Huygens RADAR team. He continues to work on radar observations of the icy satellites of Jupiter and Saturn.

Dr. Ostro was the 2003 winner of the Gerard P. Kuiper Prize from the Division of Planetary Sciences of the American Astronomical Society and has numerous publications developing the field of asteroid radar astronomy.

Ladislav Roth, photograph and biography not available at the time of publication.
Scott Shaffer, photograph and biography not available at the time of publication.

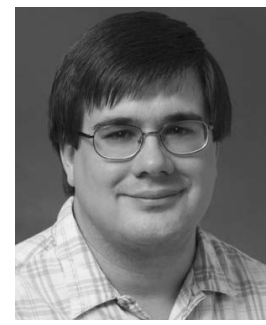

Bryan Stiles received the Ph.D. degree in electrical engineering from the University of Texas at Austin, Austin, in 1997.

Since 1997, he has been with the National Aeronautics and Space Administration's (NASA's) Jet Propulsion Laboratory (JPL), California Institute of Technology, Pasadena, where he has developed simulations, ground processing, and data analysis algorithms for spaceborne radar. He led the development of the synthetic aperture radar (SAR) processor and the point target simulator for the Cassini RADAR instrument. He has used the point target simulation to aid in the design of the instrument command sequences and pointing for the Cassini RADAR instrument. In addition to his work on Cassini, he has worked on the end-to-end simulation of ocean wind scatterometers and developed algorithms for estimating geophysical values from scatterometer data. He developed a means for improving the wind retrieval accuracy of pencil beam scanning scatterometers that is employed today by the NASA and the National Oceanic and Atmospheric Administration in processing QuikSCAT data. He also developed a statistical model of the impact of rain on ocean wind scatterometer data that is used to flag for rain contamination. He has worked on spaceborne radar systems for 10 years and Cassini RADAR for 5 years. His SAR imagery of Titan has appeared on the cover of Science. The recent publication of his work in estimating the pole and spin rate of Titan from Cassini SAR data has led to the conclusion that Titan possesses an internal ocean.

Steve Wall, photograph and biography not available at the time of publication.

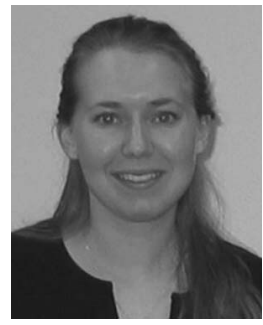

Lauren C. Wye received the B.S. degree in computer science and computer engineering with a minor in astronomy from the University of Virginia, Charlottesville, in 2003 and the M.S. degree in electrical engineering from Stanford University, Stanford, CA, in 2006, where she is currently working toward the $\mathrm{Ph} . \mathrm{D}$. degree.

She has been responsible for the Cassini RADAR scatterometer processing since the spacecraft's orbital insertion in 2004. Her research interests include radar remote sensing and planetary exploration.

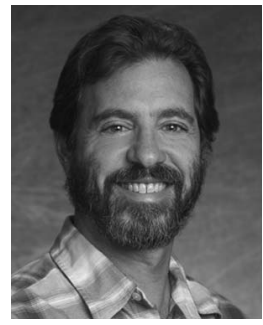

Howard A. Zebker (M'87-SM'89-F'99) received the B.S. degree from the California Institute of Technology, Pasadena in 1976, the M.S. degree from the University of California, Los Angeles, in 1979, and the Ph.D. degree from Stanford University, Stanford, CA, in 1984.

$\mathrm{He}$ is currently a Professor of geophysics and electrical engineering with Stanford University, where his research group specializes in interferometric radar remote sensing. Originally a Microwave Engineer, he built support equipment in the 1970s for the SEASAT synthetic aperture radar and designed airborne radar systems, and later developed imaging radar polarimetry, a technique for measurement of the complete scattering matrix describing a surface. He is best known for the development of radar interferometry, leading to spaceborne and airborne sensors capable of measuring topography to meter-scale accuracy and surface deformation to centimeter scale. He applies these and other processing techniques to radar remote sensing problems. Current research interests include planetary exploration, remote sensing applications, radar techniques, electromagnetic propagation and scattering, and digital signal processing. 